\title{
Effects of symmetry, methyl groups and serendipity on intramolecular vibrational energy dispersal
}

\author{
William D. Tuttle, Adrian M. Gardner, Laura E. Whalley, David J. Kemp and Timothy G. Wright ${ }^{\mathrm{a}}$
}

School of Chemistry, University of Nottingham, University Park, Nottingham NG7 2RD, UK

a Tim.Wright@nottingham.ac.uk

\begin{abstract}
We consider two key parameters that have been proposed to be important for vibrational energy delocalization, closely related to intramolecular vibrational redistribution (IVR), in molecules. These parameters are the symmetry of the molecule, and the presence of torsional (internal rotor) modes of a methyl group. We consider four para-disubstituted benzene molecules and examine their vibrational character. The molecules selected are para-difluorobenzene, para-chlorofluorobenzene, para-fluorotoluene, and para-xylene. This set of molecules allows the above parameters to be assessed in a systematic way. The probe we use is zero-electron-kinetic-energy (ZEKE) spectroscopy, which is employed in a resonant scheme, where the intermediate levels are selected vibrational levels of the $S_{1}$ excited electronic state, with wavenumbers up to $1300 \mathrm{~cm}^{-1}$. We conclude that symmetry, and the presence of a methyl groups, do indeed have a profound effect on "restricted" IVR at low energies. This is underpinned by serendipitous coincidences in the energies of the levels, owing to small shifts in vibrational wavenumbers between molecules, so bringing levels into resonance. Additionally, methyl groups play an important role in opening up new routes for coupling between vibrations of different symmetry, and this is critical in the transition to "statistical" IVR at lower energies for molecules that contain them. Further, the presence of two methyl groups in the symmetrically-substituted $p$-xylene causes more widespread IVR than does the single methyl group in the asymmetrically-substituted $p$-fluorotoluene.
\end{abstract}




\section{Introduction}

The dispersal of energy through a molecule via its vibrations can be a valuable aid to increasing its stability following the input of localized energy, such as following photoexcitation or as the result of the formation of a chemical bond. ${ }^{1,2,3}$ This process is often termed intramolecular vibrational redistribution (IVR). ${ }^{4,5,6,7,8,9,10,11,12}$ If the localized energy cannot rapidly be dispersed, then the nascent chemical bond may simply break again in a biomolecular reaction, reducing the reaction efficiency; while subsequent to photoexcitation, the molecule may photodissociate if the energy remains localized. The latter process often leads to radical products that can be involved in subsequent chemistry, and in biological systems can be very harmful, leading to photodamage and cancer initiation. ${ }^{13}$ These ideas also feed into ideas of controlling the outcomes of chemical reactions via the injection of localized energy, and has recently been discussed in relation to interactions of vibrationally-excited species with surfaces. ${ }^{14}$ If rapid dispersal of initially localized energy occurs, then it may be that the outcomes of reactions are driven simply by the total amount of energy - i.e. the Rice-Ramsperger-Kassel-Marcus (RRKM) regime ${ }^{15}$ - rather than the specific type and location of the energy; ${ }^{16}$ this has impacts - for example, Polanyi's rules ${ }^{17}$ may become inapplicable.

The design of molecules that have photophysical characteristics that enable them to disperse energy quickly is important, such as in the development of more-efficient sun protection products. ${ }^{13,18,19}$ Much ongoing work has focused on the coupling between electronic states to provide routes for energy relaxation via conical intersections; however, within the electronic states, the delocalization of energy through the chemical bond network is also of importance. ${ }^{20,21}$ The relevant motions for this are vibrations, and (where they exist) torsions. ${ }^{21,22,23,24,25}$ In all cases, the critical aspect is the coupling between these modes, with anharmonicity and vibration-torsional coupling being the principal facilitators for the wavenumber range studied for the present molecules, although rotations can also be important in IVR, ${ }^{6,9,10,26,27,28,29}$ particularly in thermal samples. ${ }^{30}$

Trying to uncover general mechanisms and aspects of molecular structure that are important in making energy dispersal efficient, are clearly important in being able to establish principles for molecular design. ${ }^{19}$ As a consequence, a number of research groups have studied families of molecules, where the molecular structure is systematically changed, and its effect on vibrational energy redistribution investigated. Since many biological molecules contain a phenyl ring, which often acts as the chromophore, then work on substituted benzenes is particularly pertinent. Of importance here, are the studies comparing IVR processes in para-difluorobenzene ( $p D F B$ ) and para-fluorotoluene $(p \mathrm{FT})$ by Parmenter and coworkers, ${ }^{28,31,32}$ with a related study by Zewail and coworkers. ${ }^{33}$ Although there has been some uncertainty regarding the levels excited (see discussion in Ref. 30), the key 
finding was that IVR was orders of magnitude faster in $p \mathrm{FT}$ than it was for corresponding vibrational levels in $p D F B$. Although initial work suggested that rotational levels were the cause of this "acceleration" of IVR, ${ }^{28}$ later work focused on the role of the methyl group, ${ }^{32,34}$ with the mode of interaction being via van der Waals interaction with $\mathrm{C}-\mathrm{H}$ bonds of the phenyl rings. ${ }^{35}$ Further work by Parmenter et al. has considered vibration-torsional levels explicitly, $36,37,38$ and our recent work showed how the methyl group was facilitating coupling between vibrational levels of different symmetry. ${ }^{39}$ Interestingly, Abel and coworkers ${ }^{40}$ studied IVR of molecules in raised-temperature, gaseous samples and concluded that the methyl group does not accelerate IVR, in contrast to low-pressure, or jetcooled gas phase studies; further comments on IVR in solution are provided in a review by the same group. ${ }^{41}$ However, note that in these ultrafast experiments a vibrational wavepacket is excited, rather than an eigenstate (see later discussion). In addition, knowledge of how solvents affect vibrational energy eigenstates in a stepwise fashion would be highly desirable in understanding such processes in more detail. Some recent work in this area has been published by Chakraborty et al. on phenol and $p$-fluorophenol complexes..$^{42,43}$

Only occasionally remarked on in previous work, ${ }^{32}$ is that as well as adding a methyl group when moving between $p \mathrm{DFB}$ and $p \mathrm{FT}$, there is also a change in symmetry between these two molecules: this can be viewed as a $D_{2 h} \rightarrow C_{2 v}$ change in point group symmetry, if the methyl group is considered as a point mass. An asymmetric substitution is expected to increase the likelihood of coupling, but a separation of the effects that are caused by the addition of the methyl group and those caused by a lowering of symmetry is not transparent. In addition to this, different substituents will lead to alterations in the vibrational wavenumbers, and can also lead to an increase in the number of vibrations; these could lead to changes in the density of states. These aspects were considered in Ref. 32, but concluded not to be a major factor in accelerating IVR. Of note, however, is that in Ref. 24 it was concluded that in the low-wavenumber regime, the occurrence of restricted IVR is critically dependent on these small changes in vibrational wavenumbers, since they move vibrations in and out of resonance. In addition, the efficiency of coupling of a particular mode to other states has also been noted to be of importance. . $4,30,44^{-}$

The role of particular vibrations in promoting IVR has been investigated by Perry et al., ${ }^{45,46}$ who suggested that modes that are "close to the centre of flexibility (COF)" accelerate IVR, with an internal rotor being such a COF. This idea clearly relies on the localization of vibrational modes, and so would refer to vibrations that include motion of the bonds closest to the $\mathrm{C}-\mathrm{CH}_{3}$ bond in molecules with a methyl group, and indeed Davies et al. ${ }^{24}$ have discussed this in their study of toluene, toluene- $\alpha-d_{3}$ and $p \mathrm{FT}$. Discussions have also been presented concerning vibrations that interact with the methyl 
group by virtue of out-of-plane motion of adjacent $\mathrm{C}-\mathrm{H}$ bonds; ${ }^{31,35,44}$ these can be thought of as "wafting" the $\mathrm{CH}_{3}$ via van der Waals interactions, which allow vibrational and torsional motion to mix. Parmenter and coworkers have commented on the out-of-plane $D_{20}$ mode ${ }^{31}$ and modelled such interactions in a qualitative manner, ${ }^{35}$ and such a model has been utilized by Davies et al. ${ }^{44}$ recently. Martens and Reinhardt ${ }^{47}$ have considered a classical model whereby low-wavenumber vibrations interact with the methyl group; they concluded that this led to chaotic effects, which are separate from the high-wavenumber vibrations.

We also note that a number of studies have tried to address the issue of the effect of symmetry on IVR. ${ }^{48,49}$ For example, von Benten et al..$^{50}$ studied benzene, benzene- $d_{1}$ and the three constitutional isomers of difluorobenzene. They concluded that for both sets of molecules, lowering the symmetry led to an increase in the rate of IVR. Experiments were carried out in 0.5 bar of the vapour in a cell at an elevated temperature, with the excitation being a femtosecond IR laser. It is unclear, however, what explicit modes were excited for each molecule in the wavepacket in these experiments, since the $60 \mathrm{fs}$ pulse employed had a width of $\sim 300 \mathrm{~cm}^{-1}$; however, these are unlikely to have been identical.

In the present work, we select four para-disubstituted benzenes, in order to gain control over the variation in parameters that change between them. These molecules are: pDFB, parachlorofluorobenzene ( $p$ CIFB), $p$ FT and $p$-xylene ( $p$ Xyl). We briefly describe the symmetry changes in these species first of all - see Figure 1. Between $p$ DFB and $p$ CIFB the point group symmetry lowers from $D_{2 h}$ to $C_{2 v}$, and a corresponding change occurs between $p \mathrm{Xyl}$ and $p \mathrm{FT}$, if the methyl groups are treated as point masses. However, if the methyl groups are explicitly considered, then one can also consider the molecular symmetry group (MSG) changing from $G_{72}$ to $G_{12}$ between the latter two molecules. Alternatively, one can view the change between $p \mathrm{DFB}$ and $p \mathrm{FT}$ and between $p \mathrm{CIFB}$ and $p \mathrm{FT}$ as the addition of a methyl group, with the point group symmetry being maintained in the latter case, again, if the methyl group is treated as a point mass. Between $p F T$ and $p X y l$ the switch of a fluorine atom to a further methyl group occurs and we move from an asymmetrically- to a symmetrically substituted molecule. One can also note that between $p D F B$ and $p X y l$ two methyl groups have replaced both fluorine atoms, with the point group being maintained as $D_{2 h}$, within the assumption of point masses for the methyl groups.

Of course, as was noted above, changing the substituents will result in corresponding vibrations changing their vibrational wavenumber, although the form of the vibration is expected to remain largely the same for most vibrations - see Ref. 51 . The small changes in form that do occur, lead to relatively minor changes in vibrational wavenumbers, but this is sometimes enough to cause the movement of levels in and out of resonance (see Refs. 24, 30 and later discussion herein). 
In the present work, we investigate the extent of IVR for various vibrational levels from the zero-point level up to levels $<1300 \mathrm{~cm}^{-1}$. Our probe is the vibrational activity excited upon ionization, monitored by zero-electron-kinetic energy (ZEKE) spectroscopy, when using a selected vibrational level in the $\mathrm{S}_{1}$ electronically-excited state as an intermediate resonance. In Figure 2, we show vibrationally-resolved resonance-enhanced multiphoton ionization (REMPI) spectra of the $S_{1} \leftarrow S_{0}$ transition for the four subject molecules, where various vibrations are indicated. We compare and contrast the activity between these vibrations for the four subject molecules, and also compare to the ZEKE spectra via the vibrationless origin. We have reported REMPI and ZEKE spectra for $p \mathrm{FT}^{24,30,39,52,53,54}$ and $p \mathrm{ClFB}^{55,56}$ and $\left.p X y\right|^{57,58}$ previously, where the assignments are discussed in detail. The spectra of $p D F B$ were recorded for the present work, and although two of the $p$ DFB ZEKE spectra to be discussed have been reported and assigned previously by Müller-Dethlefs and coworkers, ${ }^{59,60}$ the details are hard to discern in those published images.

\section{Experimental}

In previous work, cited above, we have discussed the methods employed in recording the REMPI and ZEKE spectra of $p \mathrm{ClFB}, p \mathrm{FT}$ and $p \mathrm{Xyl}$. The spectra reported herein have been recorded using the same methods and so these are only very briefly covered here.

The REMPI and ZEKE apparatus employed has been described previously in detail elsewhere. ${ }^{52,61}$ In each case, the vapour above a room temperature sample was seeded in 1.5 bar of Ar and the gaseous mixture passed through a General Valve pulsed nozzle ( $750 \mu \mathrm{m}, 10 \mathrm{~Hz}$, opening time of 180-210 $\mu \mathrm{s}$ ) to create a free jet expansion. The excitation laser was a dye laser (Sirah Cobra-Stretch) operating with C540A or C503, depending on the molecule and energetic region of interest. The ionization laser was a dye laser (Sirah Cobra-Stretch) operating with C540A, C503 (for pDFB and pClFB), DCM or Pyromethene 597 (for $p$ Xyl and $p F T$ ). The two dye lasers were pumped by either a Surelite I or Surelite III Nd:YAG laser, which varied in different experiments. For the C540A and C503 laser dyes, the third harmonic (355 nm) was used to pump the dye laser, and for the DCM and Pyromethene 597 laser dyes, the second harmonic (532 nm) was used to pump the dye laser. The fundamental frequencies produced by each dye laser were frequency doubled using BBO and KDP crystals as appropriate.

The focused, frequency-doubled outputs of the two dye lasers were overlapped spatially and temporally and passed through a vacuum chamber coaxially and counterpropagating. Here, they intersected the free jet expansion between two biased electrical grids located in the extraction region of a time-of-flight mass spectrometer, which was employed in the REMPI experiments; these grids were also used in the ZEKE experiments by application of pulsed voltages, giving typical fields $(F)$ of 
$\sim 10 \mathrm{~V} \mathrm{~cm}^{-1}$, after a delay of up to $2 \mu \mathrm{s}$, where this delay was minimized while avoiding the introduction of excess noise from the prompt electron signal. Bands had widths of $\sim 5-7 \mathrm{~cm}^{-1}$, even when $\sqrt{ } F$ relationships would suggest the widths should be significantly greater, because of the well-known decay of the lower-lying Rydberg states accessed in the pulsed-field ionization process. ${ }^{62}$

\section{Results and Discussion}

\section{Nomenclature and IVR}

In the REMPI experiments, we are probing the $\mathrm{S}_{1} \leftarrow \mathrm{S}_{0}$ electronic transition, while in the ZEKE experiments we are probing the $\mathrm{D}_{0}{ }^{+} \leftarrow \mathrm{S}_{1}$ transition, with $\mathrm{D}_{0}{ }^{+}$labelling the ground electronic state of the cation. We shall employ the $D_{i}$ labels of Ref. 51 for the vibrations, and generally refer to the symmetry of vibrations using the point group symmetry classes - the reader is referred to that work for the form of each of the vibrations. Since we employ free-jet expansions, the molecules will initially be almost exclusively in their zero-point vibrational energy level of the $S_{0}$ ground electronic state. Because of this, and since we shall explicitly note the $S_{1}$ intermediate level we employ for the ZEKE experiments, we shall usually omit the lower vibrational level when specifying transitions. Later, we shall need to refer to the torsional levels of $p \mathrm{FT}$ and $p X y l$, where we use a single, $m$, or pair, $\left\{m_{1}, m_{2}\right\}$, of quantum numbers to label these, respectively - see Ref. 57 for more details, note that the molecules will not all be in their lowest torsional level because of nuclear spin effects - see later. In addition, when vibration-torsional (vibtor) levels arise from vibration-torsional coupling, then both the vibrational and torsional quantum numbers will be specified.

When discussing IVR, the non-interacting levels are termed zero-order states (ZOSs); ${ }^{8}$ these ZOSs can be torsional or vibrational, while the levels that arise from interaction between these are termed vibrational (or vibtor) levels. Felker and Zewail et al. ${ }^{4,5,6,7,63}$ have argued that there are three regimes of IVR, covering non-existent, restrictive and dissipative (or statistical), and discuss these in the context of anthracene and deuterated anthracene, noting also that these regions have been identified in other molecules, such as azulene. ${ }^{64}$ As we shall see below, the set of molecules we have chosen exhibit each of these regimes. If two ZOSs (of whatever type) interact to form two eigenstates, then this is termed a Fermi resonance (FR), while if a small number of ZOSs interact, this can be termed a "complex Fermi resonance"; each are examples of restricted IVR. In an electronic transition, often only one of the coupled ZOSs is "bright", i.e. carries oscillator strength and as such it is only by virtue of the interactions that other ZOSs are seen - this leads to the concepts of zero-order bright (ZOB) states and zero-order dark (ZOD) states. (Note that whether a ZOS is "bright" or "dark" will depend on the type of experiment being undertaken.) If there are many ZOD states coupled to the ZOB state, then they 
are termed "bath states". There is an interesting regime when only one or two ZOD states are coupled to the ZOB state strongly, but these also couple efficiently to the bath states. This gives rise to the "tier model" (see Refs. 4 and 8), with the strongly-coupled ZOD states being termed "doorway states".

We note that vibrational ZOSs can be thought of as "diagonally" anharmonic, but it is the result of "off-diagonal" anharmonicity that leads to interactions between ZOSs. Further, in the absence of photophysical effects, such as internal conversion and intersystem crossing, the eigenstates will have a constant population - the idea of IVR comes from time-resolved experiments where a number of eigenstates are excited coherently in a wavepacket, and the changing phases of these gives the appearance of population changes.

The impact of symmetry is clearly related to the density of states (DOS), with higher symmetries expected to lead to lower DOSs for each of their particular symmetry classes. At what energies the IVR regimes occur for a particular molecule will depend on the build-up of the DOS, and we emphasise that in the lower wavenumber regions, this is expected to be erratic (see later), but will become increasingly smooth and monotonic at higher wavenumber.

In previous work, we have discussed the phenyl-localized vibrations of para-disubstituted benzenes in detail $^{51}$ and noted that there is very little change in the form of these vibrations across all common substituents. As such, we have put forward a scheme, used herein, that gives these vibrations the same label across different molecules - see Table 1. However, there are a few cases where some caution is merited, particularly when switching between a symmetrically-substituted molecule and an asymmetric one, such as between $p D F B$ and $p F T$. For example, in the symmetrically-substituted $p D F B$ molecule, the $D_{5}$ and $D_{6}$ vibrational modes contain in-phase and out-of-phase stretches of the C-F bonds, while in an asymmetrically-substituted species such as $p \mathrm{FT}$, the $D_{6}$ mode is largely a $\mathrm{C}-\mathrm{CH}_{3}$ stretch, while the $D_{5}$ mode is largely a C-F stretch, and both are of $a_{1}$ symmetry, if $C_{2 v}$ symmetry is assumed. ${ }^{51}$ In contrast, the symmetry of the asymmetric $D_{6}$ vibration in $p$ DFB is $b_{1 \mathrm{u}}$ and so is different from that of the symmetric $D_{5}$ vibration $\left(a_{g}\right)$. These differing symmetries may be expected to affect the allowed vibrational interactions and also the Franck-Condon activity in transitions. We shall comment on this further, in the below.

The interpretation of the presented spectra relies on the fact that that the ionization process is fast, and so a ZEKE spectrum generally gives a picture of the populated intermediate $\mathrm{S}_{1}$ level(s) as mapped onto the cation eigenstates. (The effect of the laser pulse duration and power density on IVR experiments has been discussed in Ref. 30.) In cases where the intermediate level has a single ZOS contribution, then the ZEKE spectrum is expected to be well-resolved, generally with a strong $\Delta v=0$ 
band, together with associated Franck-Condon (FC) active bands. The latter arise from both geometry changes between the $S_{1}$ state and the cation, but can also occur if the forms of the vibrations changes between levels (i.e. Duschinsky rotation); it is also the case that some Herzberg-Teller bands are active. If, however, the intermediate level arises from a FR between two ZOSs then, for the nanosecond laser pulses used herein, the ZEKE spectrum will appear as a juxtaposition of the expected individual ZEKE spectra of the ZOSs. Finally, if the intermediate level is a mixture of numerous ZOSs, then the ZEKE spectrum will be a juxtaposition of all contributions from these and this would result in a ZEKE spectrum where little structure was resolvable. Time-resolved photoelectron studies ${ }^{4,5,6,7,8,9,10,11,12}$ are useful for unpicking the details of the coupling (although caution is required in their interpretation ${ }^{10}$ ); in particular, since the $t=0$ spectrum can be obtained, which will usually be a good representation of the ZOB state. Subsequently, the evolution of the spectrum can be followed as a function of time to unpick the various interactions occurring. In principle, frequency-resolved spectra (such as here) will allow the same detail to be recovered, ${ }^{8,39}$ but this is only feasible when clear structure is still seen in the spectrum.

\section{Initial comments}

In Figure 2, we show the $0-1400 \mathrm{~cm}^{-1}$ regions of the REMPI spectra of the four molecules under consideration. We have given assignments of the main bands based upon previous work (see above), noting that the low wavenumber regions of the spectra of $p \mathrm{FT}$ and $p \mathrm{Xyl}$ contain many weak bands arising from torsional and vibtor levels.

In Figures 3-5 we show a series of ZEKE spectra for the four molecules, $p$ DFB, $p$ CIFB, $p F T$ and $p X y l ;$ in each case, exciting through the same intermediate level: $S_{1} 0^{0}, S_{1} 9^{1}$ and $S_{1} 5^{1}$, in order of increasing energy. (Note that we show the form of the relevant vibration in Figures 4 and 5.) As we can see from Figure 3, all four of the ZEKE spectra obtained when exciting via $\mathrm{S}_{1} 0^{0}$ have well-defined bands, with a flat baseline between all of the features. We note that several totally-symmetric vibrations are active across the spectra, allowing their wavenumbers to be established (see Table 1), for $p$ Xyl a number of these will be noted again later on, in relation to other spectra, but we note here the $3^{1}$ transition is present, but within a small group of bands, and so only an approximate value is obtained for $D_{3}$ in the cation of $\sim 1634 \mathrm{~cm}^{-1}$. In Figure 4, we can see that there is essentially a flat baseline across the spectra for $p \mathrm{DFB}$ and $p \mathrm{ClFB}$, while in $p \mathrm{FT}$ and $p \mathrm{Xyl}$ there are indications of some congestion coming into the spectrum, and this is a little more pronounced for $p$ Xyl than $p F T$. Finally, in Figure 5, we see again, essentially flat baselines, with well-defined bands for $p$ DFB and $p$ CIFB, but there is a significant amount of congestion in the $p \mathrm{FT}$ spectrum, and this is extremely pronounced in the case of $p \mathrm{Xyl}$. 
Our interpretation of the series of ZEKE spectra in Figures 3-5 is that vibrational interactions are minimal at low wavenumbers in $p D F B$ and $p$ CIFB, being localized to a few Fermi resonances, but clearly significant interactions occur in $p \mathrm{FT}$ and to a greater extent in $p \mathrm{Xyl}$, as judged by the appearance of the spectra in Figs. 4 and 5. On this basis, we can conclude that the change in point group symmetry is having a limited effect, since $p$ DFB belongs to $D_{2 h}$ and $p$ CIFB belongs to $C_{2 v}$. This is perhaps somewhat surprising, as there are almost twice as many totally-symmetric $\left(a_{1}\right)$ vibrational normal modes for $p$ CIFB than there are for $p$ DFB $\left(a_{g}\right)$. One stark manifestation of this difference is the strong $6^{1}$ transition in $p$ CIFB that is absent in $p \mathrm{DFB}$ (since the $D_{6}$ vibration is of $b_{1 u}$ symmetry in the latter molecule). Since, interactions will occur between fundamentals, overtones and combination levels then it is the buildup of the latter two type of levels that is expected to drive the extent of vibrational coupling.

If we regard the $\mathrm{CH}_{3}$ group as a point mass, then both $p \mathrm{FT}$ and $p \mathrm{ClFB}$ belong to the $C_{2 v}$ point group, and $p$ CIFB has many of its vibrations at a lower wavenumber than those in $p \mathrm{FT}$, yet $p \mathrm{FT}$ shows much more extensive IVR than does $p$ CIFB. (While it is true there are eight extra vibrations in $p$ FT from the methyl group, as well as the torsion, there is not expected to be any significant effect from these, largely since most of these are of a high wavenumber. ${ }^{32}$ ) In a similar vein, $p$ DFB and $p X y l$ each have $D_{2 h}$ point group symmetry, again if we regard the $\mathrm{CH}_{3}$ groups as point masses, and these will have similar vibrational wavenumbers as the masses of $\mathrm{F}$ and $\mathrm{CH}_{3}$ are similar. ${ }^{51}$ Again, there are additional vibrational modes (sixteen extra) from the methyl groups, but again, by analogy with $p F T$, these are not expected to have a significant effect in this wavenumber range. Despite this, it is clear that the interactions in the symmetrically-substituted $p X y l$ molecule are significantly greater than that in the symmetrically-substituted $p \mathrm{DFB}$; further, these are greater than the asymmetrically-substituted $p \mathrm{FT}$ and $p$ ClFB molecules. Clearly, other factors than simply vibrational interactions need to be considered, and the main factor will be shown to be vibrational-torsional coupling.

\section{Vibration-torsional coupling}

The possible role of vibration-torsional coupling in promoting IVR was put forward by Parmenter and coworkers some time ago, ${ }^{31}$ but this was more by implication after concluding that other factors were unlikely to be playing a key role in the increased IVR in $p$ FT compared to $p D F B .{ }^{32}$ More explicit evidence that vibration-torsional coupling is prevalent in substituted benzenes containing a methyl group was put forward by Lawrance and coworkers in their detailed studies on toluene using two-dimensional laser-induced fluorescence (2D-LIF), ${ }^{22,25}$ and these ideas have also been employed in the interpretation of time-resolved photoelectron studies of IVR ${ }^{23,24}$ and in our ZEKE $24,30,39,52,53,54$ and 2D-LIF ${ }^{39}$ studies. Of particular note is that in the work of Lawrance and ourselves on toluene and $p \mathrm{FT}$, vibtor levels have been assigned that may be viewed as combination bands of vibrational and torsional states, which are 
symmetry-allowed under the $G_{12}$ MSG, and these can be treated as ZOSs. Further, ZOSs of the same MSG symmetry class can interact, in an analogous manner as FR for pure vibrational levels. These interactions lead to mixings between the levels, causing bands to have unexpected intensities, and also be in shifted positions. In a tour de force, a comprehensive deperturbation analysis was achieved for toluene ${ }^{22}$ and the same ideas have been applied to $p \mathrm{FT} .{ }^{65}$

In very recent work, ${ }^{39}$ we provided direct evidence for coupling between vibrations of different symmetry in $p \mathrm{FT}$, which was facilitated by vibration-torsional mixing. Owing to the lowest nuclear spin states having different symmetry, it is not possible to cool the population of the $m=1$ torsional level into the $m=0$ level via collisions, and both survive in the jet expansion and so may be termed "cold" torsional levels (where $m$ is the torsional quantum number). ${ }^{22,57}$ This means transitions can occur out of either of these torsional levels, and we noted in Ref. 39 that this opens up the possibility of observing interactions that involve both $a_{1}$ and $a_{2}$ vibrations for the $m=1$ level, and similarly for $b_{1}$ and $b_{2}$ vibrations; further, Herzberg-Teller (HT) coupling then opens up the possibility for all $C_{2 v}$ classes of vibration to interact. By considering all vibrational and vibtor levels in the relevant wavenumber region, we were able to show that there were many more routes for the $2 D_{18} m=1$ vibtor level to interact with other ZOSs, than the (torsionless) $2 D_{18} \mathrm{~m}=0$ level. Thus, this showed direct evidence for how the methyl group was facilitating interactions between vibrations in this molecule; particularly noting coupling of $b_{1}$ symmetry combination vibrations (ZOD states) with an $a_{1}$ symmetry overtone (the ZOB state). Further, it was clear from the analysis that there were very few totally symmetric pure vibrational levels that could interact with the $D_{18}$ overtone, and it was the role of the methyl group in both the relaxation of the symmetry criteria for coupling vibrations and the increase in the DOS that caused the dramatic increase in coupling. ${ }^{39}$

The results shown in Figures 3-5 clearly show that the effect of point group symmetry lowering on moving from $p$ DFB to $p$ CIFB is small and confirm that the dramatic rise in IVR between $p$ DFB and $p F T$ is not a point group symmetry effect, nor a result of small changes in ring-localized vibrations, but a direct effect of the presence of the methyl group.

We now extend these arguments to the comparison of $p \mathrm{DFB}, p \mathrm{FT}$ and $p \mathrm{Xyl}$. Since the point group symmetry of both molecules is the same (considering $\mathrm{CH}_{3}$ as a point mass) and the masses of $\mathrm{CH}_{3}$ and $\mathrm{F}$ are quite similar, then one might have expected the extent of vibrational coupling in both species also to be similar. Notably, it is initially surprising that coupling in $p X y l$ is significantly more pronounced than in $p \mathrm{FT}$, since the higher (effective) point group symmetry of the former might be expected to lead to reduced vibrational coupling, as there would be fewer $a_{\mathrm{g}}$ modes in the former than $a_{1}$ modes in the 
latter. That this coupling scenario is far from the case may be rationalized by invoking vibrationtorsional coupling.

In Ref. 57, we discussed the torsional levels of $p X y l$ in detail, in terms of the $G_{72}$ MSG. For this molecule, we label each torsional level with two quantum numbers, one for each $\mathrm{CH}_{3}$ group; levels can thus be designated $\left\{m_{1}, m_{2}\right\}$, with $\{0,0\}$ being the lowest, torsionless level; additionally, there may be superscripts to indicate particular combinations of levels. ${ }^{57}$ We also showed that the lowest four torsional levels, $\{0,0\},\{0,1\},\{1,1\}$ and $\{1,-1\}$ had different nuclear spin symmetries and hence all should remain populated in the jet expansion, and hence may be termed "cold" torsional levels. In principle, therefore, excitations can occur from any of these, and we labelled the REMPI spectrum in Ref. 57 indicating this. (For clarity, in the REMPI spectrum in Figure 2, here, we have omitted much of the details of the torsional levels.)

In Table 2, we show the MSG symmetries of the vibtor levels that result when the symmetry of each of the abovementioned four torsional levels is combined with the symmetries of the different vibrations. We note that $a_{\mathrm{g}}$ vibrational modes can only interact with each other in a pure vibrational picture; however, once we include vibration-torsional coupling, then the possibilities for interaction increase markedly. For example, vibtor levels formed from $a_{\mathrm{g}}$ vibrations with the $\{0,1\}$ torsional level are able to combine with vibtor levels formed from the same torsional level, but with vibrations of $b_{1 g}$, $a_{u}$ and $b_{1 u}$ symmetries- all being of $g^{\prime \prime}$ symmetry in $G_{72}$. In fact, this means that the set of vibrations that can couple in $p \mathrm{Xyl}$ is the same as that in $p \mathrm{FT}$; additionally, further pairwise couplings are possible via the $\{1,1\}$ and $\{1,-1\}$ torsions. Taken together, we can see that the vibtor levels of an $a_{\mathrm{g}}$ vibration can facilitate interactions with vibtor levels involving other vibrations of $b_{1 g}, a_{u}$ and $b_{1 u}$ symmetry in a number of ways. Further, we know HT interactions occur, which allow $b_{3 \mathrm{~g}}$ vibrations to be active in the $\mathrm{S}_{1} \leftarrow \mathrm{S}_{0}$ spectrum for $D_{2 h}$ molecules such as $p$ DFB and $p \mathrm{Xyl}$, and these can similarly couple via various vibtor levels, similar to that discussed in Ref. 39 for $p \mathrm{FT}$. If vibration-torsion coupling occurs between FC- and HT-active modes, then in fact vibrations of any symmetry class can interact via at least one of these mechanisms. A further observation is that there are more than twice as many torsional levels in $p \mathrm{Xyl}$ as in $p \mathrm{FT}$, and concomitantly more vibtor levels for each vibrational level; as a consequence, these provide a commensurate increase in the number of interaction possibilities. It is thus clear why, despite the apparently more restrictive coupling of pure vibrations in $p$ Xyl over $p \mathrm{FT}$, in fact there are more than double the possibilities for coupling involving the vibtor levels. 


\section{Detailed comments on assignments}

We now move onto a discussion of the specific interactions that are occurring in the different molecules. First, we need to discuss the assignments of the ZEKE spectra presented here for $p$ DFB and pXyl.

\section{Assignment of pDFB spectra}

In Figure 6, we present six ZEKE spectra of $p$ DFB when exciting through six different vibrational levels of the $S_{1}$ electronic state (see Figure 2 for the REMPI spectrum). ZEKE spectra via the $0^{0}$ and $5^{1}$ levels have been presented before in Refs. 59 and 60 by Müller-Dethlefs and coworkers, but the others are presented here for the first time. We also note that Sekreta et al ${ }^{66}$ studied $p$ DFB by REMPI-PES, with the $0^{0}, 9^{1}, 18^{2}$ and $5^{1}$ being amongst those considered. We essentially concur with the assignments of the previous REMPI-PES and ZEKE spectra, ${ }^{59,60,66}$ but note that we have labelled the spectra with the $D_{i}$ labels used herein. (Our assignments of the vibrations of the cation are also generally consistent with those of Kwon et al., ${ }^{67}$ who recorded one-photon mass-analyzed threshold ionization (MATI) spectra of $p$ DFB.) The assignments of the spectra in Figure 6 are also consistent with the calculated wavenumbers of the cation of $p D F B,{ }^{58}$ and with the expected activity, based on the assignments of the $\mathrm{S}_{1}$ state intermediate levels recorded in fluorescence by Coveleskie and Parmenter, ${ }^{68}$ and Knight and Kable ${ }^{69}$ - with the assignments in the latter study being confirmed by dispersed fluorescence. We now briefly summarize the pertinent assignments of the ZEKE spectra in Figure 6.

As noted, the ZEKE spectrum via $\mathrm{S}_{1} 0^{0}$ has been presented and assigned previously. ${ }^{59,60} \mathrm{We}$ simply note here the expected dominance of the $\Delta v=0$ band, and the expected preponderance of activity from totally symmetric vibrations (fundamentals, overtones and combinations). Also of note is the clear presence of low-wavenumber bands of $b_{2 g}, b_{3 u}$ and $a_{u}$ symmetry, which are thought to arise from HT interactions in the cation.

We now move onto the ZEKE spectrum recorded via $S_{1} 9^{1}$, which is presented here for the first time. It shows a strong band at $437 \mathrm{~cm}^{-1}$, which is straightforwardly assigned as $11^{1}$, and so immediately it is clear that the $\Delta v=0$ propensity rule is not adhered to. The triplet of bands to higher wavenumber at $834 \mathrm{~cm}^{-1}, 857 \mathrm{~cm}^{-1}$ and $877 \mathrm{~cm}^{-1}$ may be straightforwardly assigned to $9^{1}, 29^{2}$ and $11^{1}$. A question immediately arises as to whether the corresponding vibrations are interacting, and if so, in the $\mathrm{S}_{1}$ state or the cation. We note that Coveleskie and Parmenter, ${ }^{68}$ and Knight and Kable ${ }^{69}$ suggested that the $9^{1}$ and $11^{2}$ levels were in Fermi resonance, with the possibility that the $29^{2}$ level was overlapped with the combination level, $17^{1} 19^{1}$. To gain further insight into these possibilities, we show the three ZEKE spectra in Figure 6 recorded via the $S_{1} D_{9}, 2 D_{29}$ and $2 D_{11}$ intermediate vibrational levels (see the REMPI 
spectrum in Figure 2). It may be seen that the spectrum via $29^{2}$ shows a strong $\Delta v=0$ band, a weak $9^{1}$ feature, and only an inkling of the $11^{2}$ band. The spectrum via $11^{2}$ shows non- $\Delta v=0$ behaviour, similar to that seen in the ZEKE spectrum of $p$ FT when exciting via this level, ${ }^{52,54}$ notably, both the $9^{1}$ and $29^{2}$ bands are quite weak. The absence of the $29^{2}$ in the origin ZEKE spectrum, but its reasonable intensity in the ZEKE spectrum via $D_{9}$, suggests that the $D_{9}$ and $2 D_{29}$ levels may be coupled in the $S_{1}$ state, although only weakly. However, some caution is merited in drawing conclusions from the $S_{1} 9^{1}$ ZEKE spectrum as the amount of UV is not constant across the range, and there is a drop-off in UV intensity towards the end of the presented spectra; hence, the $9^{2}$ band would be expected to have more intensity than it appears to have. This suggests that a progression is formed in the $D_{9}$ vibration, with a similar observation having been made in $p \mathrm{FT}^{54}$ Overall, we interpret these spectra as showing a weak interaction between the $D_{9}$ and $2 D_{29}$ vibrational levels in the $S_{1}$ state, and similarly between the $D_{9}$ and $2 D_{11}$ in the $S_{1}$ state. We note that the observation of the strong $11^{1}$ ZEKE band when exciting via $\mathrm{S}_{1} 9^{1}$ corresponds to similar such activity seen in the REMPI-PES study ${ }^{66}$ and in dispersed fluorescence, ${ }^{69}$ and could suggest that the atomic motions of the $D_{11}$ and $D_{9}$ modes undergo Duschinsky mixing between these electronic states. In addition, we note that when exciting via $S_{1} 29^{2}$, a new band is seen at $1032 \mathrm{~cm}^{-1}$, and if this were the $17^{1} 19^{1}$ band, then it would imply a value for $D_{17}$ in the cation of 729 $\mathrm{cm}^{-1}$, which is in fair agreement with the calculated value, and so we accept this assignment. Since there does not appear to be a "partner" REMPI band, it seems that the $29^{2}$ and $17^{1} 19^{1}$ REMPI (and LIF) bands are simply overlapping, as suggested in Ref. 69, rather than there being an interaction between the corresponding vibrational levels. Other bands in the spectra are generally straightforward to assign, and are not the focus of the present paper.

We now move onto the ZEKE spectrum recorded via the $S_{1} 18^{2}$ intermediate level. This clearly shows a strong $\Delta v=0$ band, consistent with the REMPI-PES spectrum of Ref. 66 . The assignment is consistent with a value for $D_{18}$ in the cation of $512 \mathrm{~cm}^{-1}-$ a value that is in line with the reported value of $\sim 510$ $\mathrm{cm}^{-1}$ in Ref. 66 . The rest of the spectrum consists of bands arising from totally-symmetric vibrations.

Finally, the ZEKE spectrum recorded via $\mathrm{S}_{1} 5^{1}$ is similar to that reported in Ref. 60 and closely resembles the REMPI-PES spectrum of Ref. 66 , with a strong $\Delta v=0$ band. Our assignments concur with those studies.

\section{Assignment of pXyl spectra}

In Figure 7, we show a series of ZEKE spectra recorded for $p$ Xyl with intermediate levels in the range $770-810 \mathrm{~cm}^{-1}$, with the excitation positions shown in the portion of the REMPI spectrum in the inset. In addition, in the top trace we also show the spectrum recorded via the $S_{1} 0^{0}$ level, which extends to 
higher wavenumber than the corresponding spectrum we reported earlier in Refs. 57 and 58, which focused on assignments of the $S_{1}$ levels below $600 \mathrm{~cm}^{-1}$, which consist of torsions, low-wavenumber vibrations, and vibration-torsional (vibtor) levels. ${ }^{57,58}$ We assign the spectra in Figure 7 by making reference to our previous work, but also to the calculated vibrational wavenumbers for the cation and $\mathrm{S}_{1}$ state in Table 1 (see also Ref. 58).

We commence with the ZEKE spectrum recorded at $\mathrm{S}_{1} 0^{0}+775 \mathrm{~cm}^{-1}$, which has bands at 805 and 950 $\mathrm{cm}^{-1}$. The former, slightly more intense band, is straightforwardly assigned as $9^{1}$, with the latter as $18^{2}$. The spectrum recorded at $\mathrm{S}_{1} 0^{0}+803 \mathrm{~cm}^{-1}$ looks somewhat similar, with the same two bands present, but with the $950 \mathrm{~cm}^{-1}$ band the more intense. For these two spectra, we infer that the $D_{9}$ and $2 D_{18}$ levels are in Fermi resonance, with the $S_{1}$ level at $775 \mathrm{~cm}^{-1}$ being dominated by $D_{9}$ (with the transition represented $9^{1} \ldots 18^{2}$ ), while the one at $803 \mathrm{~cm}^{-1}$ is dominated by $2 D_{18}$ (and the transition represented $\left.18^{2} \ldots 9^{1}\right)$. Bands to higher wavenumber in these spectra are largely associated with combinations bands of the main $\Delta v=0$ structure. However, it is clear that there is other structure between these bands, which is becoming broad in nature; particularly noticeable when exciting at $\mathrm{S}_{1} \mathrm{O}^{\circ}+803$ and $\mathrm{S}_{1} \mathrm{O}^{0}$ $+808 \mathrm{~cm}^{-1}$. When exciting at $\mathrm{S}_{1} 0^{0}+808 \mathrm{~cm}^{-1}$, again the spectrum is very different and the underlying broad, structure is becoming dominant. We see two clear bands at 884 and $950 \mathrm{~cm}^{-1}$, with the former being assigned as $11^{2}$ and the latter being $18^{2}$. We can see that the $11^{2}$ band also appears when exciting at $\mathrm{S}_{1} 0^{0}+803 \mathrm{~cm}^{-1}$, but is at most extremely weak at $\mathrm{S}_{1} 0^{0}+775 \mathrm{~cm}^{-1}$. These three spectra suggest that: the $2 D_{11}$ and $2 D_{18}$ levels are interacting significantly (a Darling-Dennison resonance ${ }^{70}$ ); the $D_{9}$ and $2 D_{18}$ levels are interacting strongly; but the $D_{9}$ and $2 D_{11}$ levels are interacting at most weakly.

We also note the appearance of a band at $990 \mathrm{~cm}^{-1}$ when exciting at $S_{1} 0^{0}+808 \mathrm{~cm}^{-1}$. A possible assignment for this band is to $16^{1} 19^{1}$, which would suggest a value for $D_{16}$ in the cation of $759 \mathrm{~cm}^{-1}$ and in the $S_{1}$ state of $595 \mathrm{~cm}^{-1}$, assuming the corresponding level is resonant in $\mathrm{S}_{1}$. These are in line with the calculated values, but we consider these assignments as tentative. To higher wavenumber, we see bands that correspond to the same ones in the $\Delta v=0$ region, but in combination with $D_{11}$.

We gain insight into the activity that is underlying the main features in the $\Delta v=0$ region by examining the low-wavenumber section of the spectrum. We have noted an aspect of ZEKE spectra in several of our previous studies, whereby we see structure to lower wavenumber that arises from "components" of combination levels, and hence gives information on the make-up of combinations that are active in the $\mathrm{D}_{0}{ }^{+} \leftarrow \mathrm{S}_{1}$ transition. In cases where significant mixing between levels has occurred, loss of resolved structure in the $\Delta v=0$ region occurs, caused by the overlap of many contributions; however, we still see well-structured "component" bands to lower wavenumber, since different intermediate eigenstates arising from the same ZOSs will give activity in the same components, which then sum 
up. ${ }^{39}$ Hence, when we examine the $420-530 \mathrm{~cm}^{-1}$ region of the ZEKE spectra recorded at $\mathrm{S}_{1} 0^{0}+803$ $\mathrm{cm}^{-1}$, we see bands at $428 \mathrm{~cm}, 441 \mathrm{~cm}^{-1}, 472 \mathrm{~cm}^{-1}, 490 \mathrm{~cm}^{-1}$ and $518 \mathrm{~cm}^{-1}$, which can be assigned as $29^{1} m=\{0,3(+)\}^{+}, 11^{1}, 19^{2}$ (with a possible contribution from $\left.18^{1}\right), 11^{1} m=\{0,3(+)\}^{-}$and $18^{1} m=\{0,3(-)\}$; in addition, we see a weak band at $44 \mathrm{~cm}^{-1}$, which is assigned as $m=\{0,3(-)\}-$ see Ref. 57 . To higher wavenumber, a very weak feature attributable to the $14^{2}$ transition can be seen at $680 \mathrm{~cm}^{-1}$. A number of these bands are reminiscent of ZEKE bands seen in our earlier work, ${ }^{58}$ occurring in a complicated REMPI feature in the range $420-445 \mathrm{~cm}^{-1}$, dominated by three bands. We note that transitions involving those three levels in combination with the $D_{29}$ vibration would fall in the wavenumber region of the $9^{1}$ and $18^{2}$ bands in the $S_{1}$ state. The low-wavenumber activity thus suggests multiple contributions to the $S_{1} 770-810 \mathrm{~cm}^{-1}$ region and suggests that vibronic coupling is significant, since the combinations of the totally-symmetric levels referred to above with $D_{29}$ would be of $b_{3 g}$ symmetry in $D_{2 h}\left(a_{4}{ }^{\prime \prime}\right.$ symmetry in $\left.G_{72}\right)$, and so these could interact with $D_{9}$ and $2 D_{18}$ via $\mathrm{HT}$ coupling between vibtor levels.

When we examine the $420-530 \mathrm{~cm}^{-1}$ region of the ZEKE spectra recorded at $\mathrm{S}_{1} 0^{0}+808 \mathrm{~cm}^{-1}$, we see one discernible band at $440 \mathrm{~cm}^{-1}$, which is a $\Delta v=-1$ contribution arising from the $11^{2}$ character. It is interesting to note that when exciting at $\mathrm{S}_{1} 0^{0}+775 \mathrm{~cm}^{-1}$ we see weaker bands at $440 \mathrm{~cm}^{-1}, 472 \mathrm{~cm}^{-1}$, $486 \mathrm{~cm}^{-1}, 518 \mathrm{~cm}^{-1}$ and $680 \mathrm{~cm}^{-1}$, with the $440 \mathrm{~cm}^{-1}$ and $472 \mathrm{~cm}^{-1}$ bands being the most prominent. The activity of these two bands, assigned to $11^{1}$ and $18^{1}$, are consistent with the assignments of the $\Delta v=$ 0 region when exciting across the $S_{1} 770-810 \mathrm{~cm}^{-1}$ range. In all spectra, the weaker features to higher wavenumber are combinations involving $D_{11}$ and the main $\Delta v=0$ bands: $9^{1} 11^{1}, 11^{3}$ and $11^{1} 18^{2}$.

Two ZEKE features appear at $\sim 850 \mathrm{~cm}^{-1}$ and $870 \mathrm{~cm}^{-1}$ when exciting via $\mathrm{S}_{1}+808 \mathrm{~cm}^{-1}$. The latter is consistent with a contribution from $17^{1} 19^{1}$; it seems unlikely that this arises from a coincident resonance in the $S_{1}$ state, since this would imply a value for $D_{17}$ in the $S_{1}$ state of $595 \mathrm{~cm}^{-1}$, which seems too far from the calculated value, and so we assume that the appearance of the ZEKE band is from FC activity. The assignment of a contribution from $18^{1} 29^{1}$ to the $850 \mathrm{~cm}^{-1}$ band is consistent with both $\mathrm{S}_{1}$ and $\mathrm{D}_{0}{ }^{+}$wavenumbers, which would be of $a_{u}$ symmetry, but can become active via vibration-torsional coupling. This assignment also seems to fit the corresponding distinct feature in the otherwise unstructured ZEKE spectrum recorded when exciting at $S_{1} 0^{0}+771 \mathrm{~cm}^{-1}$.

We now move onto the ZEKE spectra of $p$ Xyl presented in Figure 8 , recorded when exciting in the range $1140-1221 \mathrm{~cm}^{-1}$, with the excitation positions shown in the portion of the REMPI spectrum in the inset. It can immediately be seen that there is limited resolved structure in these ZEKE spectra. We first consider the spectrum recorded at $S_{1} 0^{0}+1186 \mathrm{~cm}^{-1}$. This exhibits two features: a single band at $1189 \mathrm{~cm}^{-1}$ and a double band with maxima at 1237 and $1249 \mathrm{~cm}^{-1}$. These also appear in the ZEKE 
spectrum via the origin, and can be assigned as $7^{1}, 5^{1}$ and $9^{1} 11^{1}$, respectively. The wavenumber of the intermediate level, and symmetry, suggests the main REMPI band at $1186 \mathrm{~cm}^{-1}$ arises from $5^{1}$, with the activity from $9^{1} 11^{1}$ either being FC in origin, or perhaps arises from FR in the cation; the $7^{1}$ activity appears to be FC in nature. Based on the $S_{1}$ fundamentals, the $9^{1} 11^{1}$ band would be expected at 1179 $\mathrm{cm}^{-1}$, and so this could also be present in the $S_{1}$ state, perhaps at position $\mathrm{G}\left(0^{0}+1177 \mathrm{~cm}^{-1}\right)$, and in $F R$ with the $D_{5}$ level in the $\mathrm{S}_{1}$ state.

Interestingly, when exciting at $\mathrm{S}_{1} 0^{0}+1144 \mathrm{~cm}^{-1}$, we see two clear ZEKE bands on an essentially flat baseline. The wavenumbers of $1183 \mathrm{~cm}^{-1}$ and $1326 \mathrm{~cm}^{-1}$ initially suggest assignments to $7^{1}$ and $11^{3}$, respectively, but the intensity of the latter band is not in line with FC activity and the $S_{1} 11^{3}$ level is expected at $1212 \mathrm{~cm}^{-1}$ and so is not a viable intermediate level. In addition, the $7^{1}$ transition does not appear to be active to any significant extent in REMPI spectra of the other molecules (see Figure 2 and related references). A possibility of $12^{1} 14^{1}$ was considered, but again the $S_{1}$ level is expected to be far from resonant at this wavenumber, and its intensity would be surprising for FC activity. Further consideration then led to the realization that the FR $9^{1} \ldots 18^{2} / 18^{2} \ldots 9^{1}$ REMPI bands, each in combination with $D_{29}$, would appear at $1144 \mathrm{~cm}^{-1}$ and $1178 \mathrm{~cm}^{-1}$, respectively; additionally, the corresponding ZEKE bands should appear at $1183 \mathrm{~cm}^{-1}$ and $1328 \mathrm{~cm}^{-1}$ and so are highly consistent with the two observed bands, and so we accept this assignment to a pair of FR bands from these combinations. What is remarkable is the difference in appearance of the two ZEKE spectra when exciting via the $9^{1} 29^{1}$ and $18^{2} 29^{1} \mathrm{FR}$ bands, with one being a flat baseline with distinct bands, while in the other apparently all structure is essentially lost. This suggests the latter spectrum arises from an overlap of the $18^{2} 29^{1} \mathrm{FR}$ band with another contribution: note that we mentioned above that the $9^{1} 11^{1}$ band is expected here, and so it seems that $D_{9} D_{11}$ is coupled to $D_{5}$, with the loss in structure being attributable to further coupling to other levels, which then masks the ZEKE bands arising from the $18^{2} 29^{1} \mathrm{FR}$ band.

Finally, we note that a possible assignment for the REMPI band at $1192 \mathrm{~cm}^{-1}$ that gives rise to an unstructured ZEKE spectrum would be to $6^{1} m=\{0,1\}$. If correct, it does not seem likely that this arises from direct interaction with the $D_{5} m=\{0,1\}$ vibtor levels, as this involves two fundamentals; hence, we suggest this involves a two-step (or higher) interaction. The REMPI band at $1221 \mathrm{~cm}^{-1}$ that gives rise to a ZEKE band at $1578 \mathrm{~cm}^{-1}$ appears to be consistent with an assignment to $13^{2}$ or $16^{2}$. This would giving $S_{1}$ and $D_{0}{ }^{+}$values for the fundamental of $611 \mathrm{~cm}^{-1}$ and $789 \mathrm{~cm}^{-1}$, respectively. Since we have established values for $D_{16}$ in the $S_{1}$ and $D_{0}{ }^{+}$states of $595 \mathrm{~cm}^{-1}$ and $759 \mathrm{~cm}^{-1}$, respectively (see above), we opt for the assignment of the $1221 \mathrm{~cm}^{-1}$ REMPI and $1578 \mathrm{~cm}^{-1}$ ZEKE bands to $13^{2}$. 


\section{Further comments on DOS, symmetry and IVR}

It is clear that when vibrational excitation at very high wavenumber is present in a molecule, coupling will be widespread amongst many ZOSs (statistical IVR). However, this is not true at very low wavenumber, where it is likely that interactions will only involve small numbers of vibrations (restrictive IVR). As we move up in wavenumber, the DOS will increase, but initially in an erratic matter, leading to clumps of levels. This clumpiness will be even more stark in electronic spectroscopy, since the levels observed in a spectrum by virtue of coupling will only be those localized around a ZOB state; alternatively, it is only when a ZOB state is coincident with a clump of levels that such coupling can be observed; further, it is clearly only the levels that actually couple to the ZOB state that will be seen. Ourselves, in collaboration with the Reid group, ${ }^{24,30}$ have alluded to this aspect of IVR, and we have noted this again recently. ${ }^{39}$

In the spectra shown in the present work, this localized activity is evident. For example, considering the $D_{6}$ and the $D_{5}$ vibrations. We first note that the $D_{6}$ vibration is not active in $p$ DFB or $p X y l$, since it is not totally-symmetric under $D_{2 \mathrm{~h}}$ symmetry, but the $D_{5}$ vibration is active in all four molecules. This is a good example of how the lowering of symmetry might be expected to increase coupling. When comparing the ZEKE spectra for $p \mathrm{FT}$ via each of these two vibrations, the loss of structure due to IVR is significantly more marked for $5^{1}$ than $6^{1}$, even though these two vibrations are only $36 \mathrm{~cm}^{-1}$ apart; this has been remarked on previously. ${ }^{30}$ Another example would be the set of ZEKE spectra for $p X y l$ in Figure 7, where the spectrum at $+771 \mathrm{~cm}^{-1}$ is unstructured, that at $+775 \mathrm{~cm}^{-1}$ is well structured, and then we see broad structure building up as we move through the $+803 \mathrm{~cm}^{-1}$ and $+808 \mathrm{~cm}^{-1}$ spectra. The DOS will not be increasing monotonically, nor especially rapidly, across this narrow wavenumber range, and the observed behaviour seems more likely to be associated with ZOB states hitting clumps of ZOSs at particular wavenumbers and then coupling to them. We see similar variation in structuredness as we cross the series of spectra in Figure 8, with the spectrum obtained when exciting at $\mathrm{S}_{1}+1144 \mathrm{~cm}^{-1}$ being notably simple and structured.

We now look at the DOS for the four molecules in more detail. To do this, we select a $\pm 20 \mathrm{~cm}^{-1}$ window around the respective $D_{9}$ and $D_{5}$ vibrations, and summarize in Table 3 the number of levels that we find, which can act as ZOSs. We first consider totally-symmetric vibrational levels only ( $a_{1}$ or $a_{\mathrm{g}}$ ), which would be able to couple directly to the $D_{9}$ level; we see that $p$ CIFB and $p$ DFB have 6 and 5 levels, respectively, and $p \mathrm{FT}$ has 6 ; rather surprisingly $p \mathrm{Xyl}$ has only 1 , highlighting the role of serendipity in the proximity of vibrational ZOSs at these low wavenumbers. On the other hand, when we consider all vibrational levels, the respective numbers are $30,19,14$ and 18 for $p$ CIFB, $p$ DFB, $p$ FT and $p$ Xyl respectively. We emphasise that these numbers are comparatively small, and so significant changes 
can result from just a handful of levels falling inside or outside of the selected window, which itself is dependent on the wavenumber of the $D_{9}$ vibration for a particular molecule. When we look at the higher-wavenumber $D_{5}$ vibration, we see the numbers of totally-symmetric vibrational levels are, respectively, 18, 7, 21 and 9, while the total number of vibrational levels are $85,74,91$ and 72 . Thus, overall, the numbers are larger for the higher-wavenumber $D_{5}$ vibration than for the lower $D_{9}$, as expected, but not high enough to explain the dramatic changes in the appearances of the spectra. In addition, the numbers for $p$ Xyl would not be expected to lead to the notable loss of structure observed compared to the other species.

We now include the effect of vibration-torsional coupling for $p \mathrm{FT}$ and $p$ Xyl. Table 3 also shows the total number of totally-symmetric levels arising from either vibrational or vibtor levels. These can be seen to be $6,5,17$ and 14 for the $D_{9}$ vibration of for $p$ CIFB, $p$ DFB, $p F T$ and $p X y l$, respectively. If we then include vibrational or vibtor levels that have allowed transitions from the "cold" torsional levels, the numbers become 6, 5, 47, and 169; while if we further allow HT coupling and other mechanisms that allow all of these levels to be considered as coupling to some extent, then the respective final numbers are $30,19,123$, and 595 . Thus, it becomes clear that the torsional levels do indeed lead to a dramatic increase in the DOS, and this becomes more marked as more coupling mechanisms open up. We now move to the $D_{5}$ level, where we find $18,7,76$, and 81 totally symmetric vibrational or vibtor levels; 18 , 7, 221 and 734 levels that have allowed transitions from the "cold" torsional levels; and finally overall totals of 85, 74, 610 and 2602 levels that could couple if HT coupling or other mechanisms operate. Note that we have included all phenyl-ring-localized vibrational levels up to four quanta in our analyses, and all vibtor levels arising from these vibrations, with torsional levels that are accessible from the four lowest levels with up to $\Delta m$ or $\Delta\left(m_{1}+m_{2}\right)=6$, for $p$ FT and $p$ Xyl, respectively. Note that coupling is only expected to occur between levels that have the same nuclear spin symmetry; hence, although a particular vibration may be coupled to other vibrations via interactions between vibtor levels, such interactions will split into two groups for $p F T$ and four for $p X y l$, corresponding to the nuclear spin symmetries of each of the "cold" torsional levels noted earlier, since neither electronic excitation nor the interactions between vibrational or vibtor levels can change the nuclear spin.

Clearly, the more levels that lie close to a ZOB state, the more possibilities there are for coupling, but only a small number of these levels would be expected to couple strongly (i.e. act as "doorway states"), but subsequent couplings and other multistep pathways will be present, leading to widespread interactions of many levels. The numbers noted above and summarized in Table 3, clearly show why there is more interaction between the levels of $p \mathrm{FT}$ and $p \mathrm{Xyl}$ compared to $p \mathrm{CIFB}$ and $p \mathrm{DFB}$, and further why the mixing is greater in $p X y l$ than $p F T$. An explicit example of a ZOB state in $p F T, 2 D_{18}$, that is an 
overtone of an out-of-plane vibration, being coupled to vibrations of different symmetry via vibtor levels, was discussed in Ref. 39; however, we expect such mechanisms to be widespread.

A summary of the build-up of the vibrations, according to the various criteria outlined above, is provided in Figure 9, where the plots are obtained by summing up the number of levels within $10 \mathrm{~cm}^{-1}$ windows (i.e. a DOS plot). A similar plot is presented in Figure 10, where the torsional and vibtor levels have been included for $p \mathrm{FT}$ and $p \mathrm{Xyl}$. It may be seen from Figure 9 that the DOS of totally-symmetric vibrational levels is largely the same across all four molecules. We now consider vibtor levels arising from totally-symmetric vibrations coupled to the "cold" torsional levels, and find that the number of levels that can couple to these increases dramatically for $p F T$ and $p$ Xyl (Figure 10), while (of course) this changes nothing for $p D F B$ and $p$ ClFB. When we look at the total numbers of levels of all symmetries, we can see from Figure 10 that there are strong oscillations in the DOS for both $p$ DFB and $p \mathrm{CIFB}$, while these become less pronounced moving through $p \mathrm{FT}$ and $p \mathrm{Xyl}$. These plots illustrate that the strongest couplings of $Z O B$ states are going to rely on fortuitous coincidences at low wavenumber in $p \mathrm{DFB}$ and $p \mathrm{ClFB}$, explaining why vibrational coupling there consists of the occasional FR. In contrast, even at moderately low wavenumbers, the build-up in levels for $p \mathrm{FT}$, and particularly $p X y l$, illustrates that these coincidences are much more common, indicating widespread mixing of ZOSs and hence the broad unstructured nature of the ZEKE spectra seen when exciting via $\mathrm{S}_{1} 5^{1}$ in both molecules, and even $\mathrm{S}_{1} 9^{1}$ in $p \mathrm{Xyl}$. The spectra indicate that some of these mixings are strong, and may indicate doorway state behaviour. In Figure 11, we have overlaid the corresponding REMPI spectra on the DOS plots. It may be seen that for $D_{9}$ and $D_{5}$, the transitions are only coincident with many ZOSs in the cases of $p \mathrm{FT}$ and $p \mathrm{Xyl}$.

Note that these DOS calculations have neglected the methyl-localized vibrations. None of these can couple to $D_{9}$, since they are all above $900 \mathrm{~cm}^{-1}$; however, some vibtor levels of these will be in the correct wavenumber region potentially to couple to $D_{5}$. Despite this, the number of these falling in a $\pm 20 \mathrm{~cm}^{-1}$ window is not expected to be large, and the trends we see for the $D_{9}$ vibration, for which we have noted no contribution is possible, suggests this will be a minor perturbation on the overall picture involving the DOS for $D_{5}$.

\section{General comments on IVR}

In spectroscopic studies of vibrational interactions (such as IVR) via dispersed fluorescence (DF) or photoelectron spectroscopy (PES), of which ZEKE is an example, one is projecting the $\mathrm{S}_{1}$ state level(s) onto those of the $\mathrm{S}_{0}$ state (in DF) or the $\mathrm{D}_{0}{ }^{+}$state (ZEKE). In the absence of vibronic or other interactions, and if the vibrations had identical forms in all three states, and all three states had the 
same geometry, then the DF and ZEKE spectra when exciting via a particular $\mathrm{S}_{1}$ vibrational level would comprise a single $\Delta v=0$ feature. Of course the geometries of the three states are not expected to be precisely the same, and so we expect to see vibrational activity based on the Franck-Condon principle. If we imagined a gedanken experiment where the geometry had changed, but the vibrations all had precisely the same form, then we would see clean activity, including progressions, of vibrations that corresponded to the geometry change between the two states involved; these would only involve vibrations that are totally symmetric in the point group that is common to both states. In reality, deviations from this simple picture can occur from several sources. One would be HT interactions, which (for $C_{2 v}$ symmetry) can give rise to new bands from vibrations of different symmetry (such as $b_{2}$ ), but can also affect the intensities of totally-symmetric bands. Another would be Coriolis interactions, which could also give activity in non-totally symmetric bands (but Coriolis effects are not expected to be of importance in jet-cooled studies). Another would be that the changes in geometry will, of course, give rise to changes in the forms of the vibrations, and as such the vibrational activity will alter. We can think of the vibrations in the final states as being made up of linear combinations of those in the intermediate level - i.e. Duschinsky rotation - but this is not vibrational coupling, per se. Such effects need to be considered when making conclusions from spectral activity.

In the time domain, discussion often refers to energy flowing through the molecule, populations changing, and vibrational energy becoming redistributed between modes as a function of time. However, in the frequency domain, eigenstates (strictly vibrational eigenstates, as we are ignoring rotations) will have a fixed population and the motion is well-defined. These apparently contradictory pictures are, of course, reconcilable. The issue arises from the fact that time-domain studies use picosecond or even femtosecond pulses, and these have a significant width in wavenumbers, from tens to even hundreds of $\mathrm{cm}^{-1}$. Thus, in these ultrashort laser pulse experiments, a number of eigenstates are usually excited coherently, and indeed it is this that gives rise to the observed timedependence. If one excites a single uncoupled vibrational state - for example, one that is energetically distant from any other state (i.e. it is a single ZOS) - then no time dependence would be observed whatever the pulse duration. In a similar way, if one excites a single eigenstate that that arises from the interaction of ZOSs, with an ultrashort laser pulse, then of course still no time dependence would be observed.

If we consider the simplest case where two ZOSs interact strongly, one of which is "bright" and the other of which is "dark" then we form two eigenstates, each with a significant character of each of the ZOSs. Further, if these two eigenstates are excited coherently, and with the same probability, then at $t=0$ the superposition of the two spectra from these will resemble that of the ZOB state (the ZOB 
components are in-phase, but the ZOD components are out-of-phase); however, the wavepacket will evolve, and sometime later, the superposition will resemble the ZOD state (the ZOB components are out-of-phase, but the ZOD components are in-phase), and so on - quantum beating. If we interrogate this population in an ultrashort laser experiment, by projecting the population onto the vibrational levels in another state, for example by PES, then we will see the population apparently switching between the ZOB state and the ZOD state, even though the form of the individual eigenstates and their populations are not changing. In more complicated systems, the interactions could give rise to multiple eigenstates contributing to the wavepacket and although at $t=0$ it should resemble the ZOB state closely (providing all relevant eigenstates have been excited with equal probabilities), it is possible that rapid dephasing causes any resulting spectrum to become unstructured, even at short times. Still, however, the motions and the populations of the multiple eigenstates will have remained unaltered. In the frequency domain, the idea of IVR is thus really a misnomer, since, as noted above, the populations and motions of the eigenstates are constant, even though these eigenstates could be the result of interactions between a large number of ZOSs. However, the result of such interactions is clearly a wider dispersal of the energy through the molecular degrees of freedom.

The usefulness of the time domain picture can be illustrated by a simple electronic absorption experiment. Clearly, if a ZOS is localized in nature, and poorly coupled to any other level, then its excitation will allow mode-specific excitation to be studied. ${ }^{14}$ Alternatively, if a ZOB state is coupled to a number of other ZOSs, and we excite the molecule with an ultrashort laser pulse then, in some circumstances, we will be able to create a wavepacket that resembles the ZOB state long enough that mode-selective excitation can still occur, and reactivity might be controllable. Another useful situation in which to view IVR in the time domain is when a new chemical bond is made in an isolated species, where the initial excitation is localized in the new bond. If this is an uncoupled local mode (or a weakly coupled mode), then the energy cannot be efficiently dissipated, and the bond will break again (the most obvious case of this is that of two atoms colliding to form a diatomic molecule). If, however, the new chemical bond corresponds to a ZOS that is involved in a wide range of coupled vibrational eigenstates, then the bond formation will result in the excitation of this wide range of vibrational eigenstates. Initially, the formed wavepacket will look like the localized ZOS, but the energy is actually shared between many vibrational eigenstates, and the dispersed nature of the ZOSs becomes evident as the vibrational eigenstates dephase.

The present work indicates that the presence of methyl groups in a molecule will make it more difficult to undertake vibrational state-selective experiments, and that the more methyl groups there are, the 
more unlikely it will be that selectively is achievable, even if the effective point-group symmetry (i.e. with the assumption of a point mass for the methyl group) of the molecule increases.

\section{Concluding remarks}

In this work, we have shown that in closely-related species, point group symmetry does not appear to have a large effect on IVR, which is contrary to some other studies. ${ }^{48,49,50}$ Two of these studies did not consider jet-cooled molecules, ${ }^{48,50}$ and additionally in the ultrafast study ${ }^{50}$ a $300 \mathrm{~cm}^{-1}$ wide pulse was used in creating the wavepacket, and hence in those experiments other factors are likely playing a role. In addition, two of the studies considered relatively high-lying levels, ${ }^{49,50}$ where we would expect the DOS of states to be largely continuous. As a consequence, the IVR behaviour probed in those studies is very different to that here, and illustrates the importance of considering the role of the experimental conditions on conclusions regarding IVR - see discussion in Ref. 30. At low wavenumbers, the erratic build-up of the DOS produces a role for serendipity in whether the wavenumber of a ZOB state coincides with a clump of ZOSs, and indeed whether it can couple to them. The implication of this is, of course, that one can only observe interactions between vibrational levels and, by extension, IVR, in a localized sense. As a consequence, some caution is required in making general conclusions based on a small number of observations.

On the other hand, here and in Ref. 39, we have shown that the presence of methyl groups does play a prominent role, both in the build-up of the DOS, but also in the facilitation of coupling between vibrational levels of different symmetry. Indeed, the effect of the presence of methyl groups appears to outweigh any symmetry effects. We emphasise that these coupling effects are going to be most clearly discernible at low wavenumbers, but will play a role over all wavenumber ranges. We have noted that above-cited early work by Parmenter and coworkers concluded the role of the methyl rotor was significant, and this fits the observation of vibtor states and interactions involving them, seen by our group and that of Lawrance and coworkers (see above citations). The conclusion of von Benten at al., ${ }^{40,41}$ that the presence of a methyl rotor, or $-\mathrm{CF}_{3}$ group, is irrelevant is clearly contradictory to the present and other jet studies. We ascribe this difference in conclusion to the fact that those studies ${ }^{40,41}$ were undertaken at significant gas pressures and elevated temperatures, or in solution; and, further, used ultrashort laser pulses so that single eigenstate resolution was not possible (or desirable for those experiments). As a consequence, again we emphasise that the important role played by the experimental conditions in observations on IVR, and making reliable general conclusions is often far from straightforward. 
In summary, in the present work, we have examined the ZEKE spectra of four para-disubstituted benzene molecules, when exciting via different vibrational levels of the $S_{1}$ state over a range of vibrational wavenumbers. This has allowed us to compare the effect of both the point group symmetry and the molecular symmetry group on coupling between vibrational and vibtor levels. In so doing, we have been able to isolate the point group symmetry changes from that of the addition of a methyl group. Moreover, when comparing the behaviour of a molecule with one methyl group $(p \mathrm{FT})$ with that of a molecule with two $(p \mathrm{Xyl})$, we see increased coupling even though we have moved from an asymmetrically-substituted molecule to a symmetrically-substituted one. We have associated this with the increase in the number of torsional levels, which is more than doubled via the in-phase and out-of-phase motions of the two rotors in $p X y l$. Further, the differing nuclear spin symmetries of the four lowest torsional levels means that these are all populated, even under jet-cooled conditions. In addition, any pure vibrational state gives rise to many vibtor levels, and a number of these will have symmetries that open up routes to coupling between vibrations of different symmetry, via their various vibtor levels.

The appearance of each of the $p F T$ and $p X y I$ ZEKE spectra are seen to change significantly even when exciting via levels that are close in wavenumber, implying that these have very different access to the underlying bath of states that is building up with increasing wavenumber, initially erratically, and then continuously (but not uniformly). This restricted access could be the result of weak coupling, and so a more stringent requirement on energetic proximity, and/or it could be the result of symmetry constraints. Some evidence for the serendipity of the possibility of interactions comes from noting that the $D_{9}$ vibration is coupled to $2 D_{29}$ in $p$ ClFB and $p F T$, even though one of the substituents has a significantly different mass, while the same vibration couples to the $2 D_{18}$ level in $p$ Xyl (where the $29^{2}$ band appears to be exceptionally weak or absent, suggesting little interaction). In the $p$ DFB molecule the $D_{9}$ level has been hypothesised as being coupled to $2 D_{11}$; further, the $2 D_{29}$ level is weakly coupled to $D_{9}$ in this molecule (where $2 D_{29}$ appears to be overlapped, and so apparently not interacting with, the $17^{1} 19^{1}$ level). In a similar vein, $D_{5}$ does not appear to be coupled to any level in $p D F B$ (see above, and Ref. 69), but this vibration is in FR with the $2 D_{10}$ level in $p$ ClFB; $; 5,56$ while it is clear that there is widespread coupling of $D_{5}$ in $p \mathrm{FT}$ and $p \mathrm{Xyl}$, even though there are few pure vibrational levels that are totally symmetric and energetically close.

To gain insight into this, we looked explicitly at the build-up of the vibrational and vibtor levels in the range $0-1400 \mathrm{~cm}^{-1}$ in the four molecules and have shown that this gives a clear rationale for the observed behaviour. Further, it indicates the clumpiness of the build-up of vibrational levels for $p D F B$ and $p \mathrm{ClFB}$, and the dramatic localized increases in the DOS once vibtor levels are included for $p \mathrm{FT}$ and 
$p \mathrm{Xyl}$; this build-up is more rapid for $p \mathrm{Xyl}$ than for $p \mathrm{FT}$, owing to larger number of torsional levels that are made of the different combinations of the torsional levels of each methyl group. As we explicitly saw in Ref. 39, but which we expected to be a more general picture, and is confirmed herein, the torsional levels facilitate coupling between vibrational levels of different symmetry, via the creation of many totally-symmetric vibtor levels. Together with other coupling mechanisms, such as HT and Coriolis, there are routes for vibrations of all symmetry classes to interact via their vibtor levels, but still restricted to the same nuclear spin symmetries, and hence a rapid increase in the coupling possibilities.

Overall, the general conclusion from the present work is that the presence of $\mathrm{CH}_{3}$ groups does indeed increase the propensity for IVR, and appears to override any vibrational symmetry constraints. We anticipate that these ideas will generalize, and cover other low-frequency, wide-amplitude motions, such as $\mathrm{NH}_{2}$ inversions, alkyl chain bends and so on. In particular, for long, flexible chains attached to phenyl rings, it may be anticipated that there could be interactions with the phenyl-localized out-ofplane modes. 


\section{Acknowledgements}

We are grateful to the EPSRC for funding (grant EP/L021366/1). The EPSRC and the University of Nottingham are thanked for studentships to W.D.T., D. J. K. and L.E.W. We are grateful to the High Performance Computer resource at the University of Nottingham. 
Table 1: Calculated and experimental vibrational wavenumbers for $p X y \mid$

\begin{tabular}{|c|c|c|c|c|c|}
\hline \multirow[t]{3}{*}{$D_{\mathrm{i}}$} & \multirow[t]{3}{*}{ Mulliken $\left(D_{2 h}\right)$} & \multicolumn{4}{|c|}{$p \times y l$} \\
\hline & & \multicolumn{2}{|c|}{$\mathrm{S}_{1}$} & \multicolumn{2}{|c|}{$\mathrm{D}_{0}{ }^{+}$} \\
\hline & & Calc $^{a}$ & Expt $^{b}$ & Calc $^{c}$ & Expt $^{b}$ \\
\hline \multicolumn{6}{|c|}{$a_{1}$} \\
\hline$D_{1}$ & $1\left(a_{g}\right)$ & 3105 & & 3106 & \\
\hline$D_{2}$ & $10\left(b_{1 u}\right)$ & 3088 & & 3093 & \\
\hline$D_{3}$ & $2\left(a_{g}\right)$ & 1542 & & 1633 & $\sim 1634^{d}$ \\
\hline$D_{4}$ & $11\left(b_{1 u}\right)$ & 1437 & & 1450 & \\
\hline$D_{5}$ & $3\left(a_{g}\right)$ & 1176 & $1186^{d}$ & 1229 & $1237^{d}$ \\
\hline$D_{6}$ & $12\left(b_{1 u}\right)$ & 1192 & 1192 & 1209 & \\
\hline$D_{7}$ & $4\left(a_{g}\right)$ & 1147 & & 1175 & 1189 \\
\hline$D_{8}$ & $13\left(b_{1 u}\right)$ & 959 & & 965 & \\
\hline$D_{9}$ & $5\left(a_{g}\right)$ & 784 & $775^{d}$ & 791 & 805 \\
\hline$D_{10}$ & $14\left(b_{1 u}\right)$ & 694 & & 693 & \\
\hline$D_{11}$ & $6\left(a_{g}\right)$ & 402 & 404 & 440 & 439 \\
\hline \multicolumn{6}{|c|}{$a_{2}$} \\
\hline$D_{12}$ & $7\left(a_{u}\right)$ & 653 & & 994 & \\
\hline$D_{13}$ & $9\left(b_{1 g}\right)$ & 546 & 611 & 782 & 789 \\
\hline$D_{14}$ & $8\left(a_{u}\right)$ & 201 & 217 & 349 & 340 \\
\hline \multicolumn{6}{|c|}{$b_{1}$} \\
\hline$D_{15}$ & $15\left(b_{2 g}\right)$ & 719 & 669 & 1005 & 1007 \\
\hline$D_{16}$ & $28\left(b_{3 u}\right)$ & 640 & 595 & 784 & 759 \\
\hline$D_{17}$ & $16\left(b_{2 g}\right)$ & 502 & & 641 & 639 \\
\hline$D_{18}$ & $29\left(b_{3 u}\right)$ & 443 & 400 & 470 & 476 \\
\hline$D_{19}$ & $17\left(b_{2 g}\right)$ & 203 & 213 & 232 & 231 \\
\hline$D_{20}$ & $30\left(b_{3 u}\right)$ & 104 & 106 & 100 & 102 \\
\hline \multicolumn{6}{|c|}{$b_{2}$} \\
\hline$D_{21}$ & $18\left(b_{2 u}\right)$ & 3100 & & 3104 & \\
\hline$D_{22}$ & $23\left(b_{3 g}\right)$ & 3084 & & 3092 & \\
\hline$D_{23}$ & $24\left(b_{3 g}\right)$ & 1445 & & 1399 & \\
\hline$D_{24}$ & $19\left(b_{2 u}\right)$ & 1322 & & 1470 & \\
\hline$D_{25}$ & $20\left(b_{2 u}\right)$ & 1390 & & 1300 & \\
\hline$D_{26}$ & $25\left(b_{3 g}\right)$ & 1277 & & 1250 & \\
\hline$D_{27}$ & $21\left(b_{2 u}\right)$ & 920 & & 1133 & \\
\hline$D_{28}$ & $26\left(b_{3 g}\right)$ & 545 & 554 & 553 & 555 \\
\hline$D_{29}$ & $27\left(b_{3 g}\right)$ & 367 & 370 & 376 & 378 \\
\hline$D_{30}$ & $22\left(b_{2 u}\right)$ & 283 & & 289 & \\
\hline
\end{tabular}


${ }^{a}$ TD-DFT B3LYP/aVTZ scaled by 0.97.

${ }^{b}$ Experimental values from Refs. 57 and 58, and present work - see text for details.

c UB3LYP/aVTZ scaled by $0.97 ;\left\langle S^{2}>\right.$ less than 0.76 .

${ }^{d}$ Likely in Fermi resonance - see text. 
Table 2

\begin{tabular}{|c|c|c|c|c|c|}
\hline \multicolumn{2}{|c|}{ Vib. Symm. } & \multicolumn{4}{c|}{ Vibtor Symm. } \\
\hline$D_{2 h}$ & $G_{72}$ & $\{0,0\}-\mathrm{A}_{1}{ }^{\prime}$ & $\{0,1\}-\mathrm{G}^{\prime \prime}$ & $\{1,1\}-\mathrm{E}_{3}{ }^{\prime}$ & $\{1,-1\}-\mathrm{E}_{1}{ }^{\prime}$ \\
\hline$a_{g}$ & $a_{1}{ }^{\prime}$ & $a_{1}{ }^{\prime}$ & $g^{\prime \prime}$ & $e_{3}{ }^{\prime}$ & $e_{1}{ }^{\prime}$ \\
\hline$b_{1 g}$ & $a_{2}{ }^{\prime}$ & $a_{2}{ }^{\prime}$ & $g^{\prime \prime}$ & $e_{4}{ }^{\prime}$ & $e_{1}{ }^{\prime}$ \\
\hline$b_{2 g}$ & $a_{3}{ }^{\prime \prime}$ & $a_{3}{ }^{\prime \prime}$ & $g^{\prime}$ & $e_{3}{ }^{\prime \prime}$ & $e_{2}{ }^{\prime \prime}$ \\
\hline$b_{3 g}$ & $a_{4}{ }^{\prime \prime}$ & $a_{4}{ }^{\prime \prime}$ & $g^{\prime}$ & $e_{4}{ }^{\prime \prime}$ & $e_{2}{ }^{\prime \prime}$ \\
\hline$a_{u}$ & $a_{3}{ }^{\prime}$ & $a_{3}{ }^{\prime}$ & $g^{\prime \prime}$ & $e_{3}{ }^{\prime}$ & $e_{2}{ }^{\prime}$ \\
\hline$b_{1 u}$ & $a_{4}{ }^{\prime}$ & $a_{4}{ }^{\prime}$ & $g^{\prime \prime}$ & $e_{4}{ }^{\prime}$ & $e_{2}{ }^{\prime}$ \\
\hline$b_{2 u}$ & $a_{1}{ }^{\prime \prime}$ & $a_{1}{ }^{\prime \prime}$ & $g^{\prime}$ & $e_{3}{ }^{\prime \prime}$ & $e_{1}{ }^{\prime \prime}$ \\
\hline$b_{3 u}$ & $a_{2}{ }^{\prime \prime}$ & $a_{2}{ }^{\prime \prime}$ & $g^{\prime}$ & $e_{4}{ }^{\prime \prime}$ & $e_{1}{ }^{\prime \prime}$ \\
\hline
\end{tabular}


Table 3: Numbers of vibrational and vibtor levels close to $D_{9}$ and $D_{5}$

\begin{tabular}{|c|c|c|c|c|}
\hline & $p$ ClFB & $p F T$ & $p D F B$ & $p \times y l$ \\
\hline \multicolumn{5}{|c|}{$D_{9} \pm 20 \mathrm{~cm}^{-1}$} \\
\hline $\begin{array}{l}\text { Totally-symmetric } \\
\text { vibrational levels }\end{array}$ & 6 & 6 & 5 & 1 \\
\hline $\begin{array}{c}\text { Totally-symmetric } \\
\text { vibrational and vibtor } \\
\text { levels }\end{array}$ & 6 & 17 & 5 & 14 \\
\hline Vibrational levels & 30 & 14 & 19 & 18 \\
\hline $\begin{array}{l}\text { Total that are accessible } \\
\text { from the "cold" torsional } \\
\text { levels }\end{array}$ & 6 & 47 & 5 & 169 \\
\hline Total (all symmetries) & 30 & 123 & 19 & 595 \\
\hline \multicolumn{5}{|c|}{$D_{5} \pm 20 \mathrm{~cm}^{-1}$} \\
\hline $\begin{array}{l}\text { Totally-symmetric } \\
\text { vibrational levels }\end{array}$ & 18 & 21 & 7 & 9 \\
\hline $\begin{array}{c}\text { Totally-symmetric } \\
\text { vibrational and vibtor } \\
\text { levels }\end{array}$ & 18 & 76 & 7 & 81 \\
\hline Vibrational levels & 85 & 91 & 74 & 72 \\
\hline $\begin{array}{c}\text { Total that are accessible } \\
\text { from the "cold" torsional } \\
\text { levels }\end{array}$ & 18 & 221 & 7 & 734 \\
\hline Total (all symmetries) & 85 & 610 & 74 & 2602 \\
\hline
\end{tabular}




\section{Figure Captions}

Figure 1: Structures of the molecules discussed in this work, with their point groups and, where applicable the molecular symmetry group. In designating a point group for $p \mathrm{FT}$ and $p \mathrm{Xyl}$, we are assuming the methyl group is a point mass.

Figure 2: REMPI spectra $\left(0-1400 \mathrm{~cm}^{-1}\right)$ of the four molecules discussed in this work. The assignments are discussed in references given in the text for $p$ CIFB, $p$ DFB, $p F T$ and the $0-600 \mathrm{~cm}^{-1}$ region of $p X y l$. The higher-wavenumber assignments for $p \mathrm{XYl}$ are discussed in the present work. The assignments given in red indicate bands which has been used as intermediates in recording presented ZEKE spectra - see text.

Figure 3: ZEKE spectra recorded via the origin of the $S_{1} \leftarrow S_{0}$ transition for the four molecules discussed in the present work. The assignments mainly come from previous work cited in the text - also see text for further discussion. Bands marked with an asterisk for $p$ CIFB and $p$ DFB are accidental resonances (see Refs. 56 and 60) and not part of the ZEKE spectrum.

Figure 4: ZEKE spectra recorded via the $S_{1} 9^{1} \leftarrow S_{0}$ transition for the four molecules discussed in the present work. The assignments for $p$ CIFB, $p D F B$ and $p F T$ come from previous work cited in the text also see text for further discussion. The assignments for $p$ Xyl are discussed in the text. Bands marked with an asterisk for $p$ CIFB and $p$ DFB are accidental resonances (see Refs. 56 and 60) and not part of the ZEKE spectrum. The mode diagram for the $D_{9}$ vibration from Ref. 51 is shown, and is similar for all four molecules.

Figure 5: ZEKE spectra recorded via the $S_{1} 5^{1} \leftarrow S_{0}$ transition for the four molecules discussed in the present work. The assignments for $p$ CIFB, $p \mathrm{DFB}$ and $p \mathrm{FT}$ come from previous work cited in the text also see text for further discussion. The assignments for $p \mathrm{Xyl}$ are discussed in the text. Bands marked with an asterisk for $p$ CIFB and $p$ DFB are accidental resonances (see Refs. 56 and 60) and not part of the ZEKE spectrum. Mode diagrams for the $D_{5}$ vibration from Ref. 51 are shown, which may be seen to be a symmetric stretch of both $\mathrm{C}-\mathrm{X}$ bonds $\left(\mathrm{X}=\mathrm{F}\right.$ or $\left.\mathrm{CH}_{3}\right)$ for the symmetrically substituted species, but is a localized C-F stretch for the asymmetrically substituted species - see Ref. 51 for further discussion. 
Figure 6: ZEKE spectra for $p$ DFB recorded via a series of intermediate states, whose relative wavenumbers in the $S_{1}$ state may be seen in Figure 2. Bands marked with an asterisk are accidental resonances (see Ref. 56) and not part of the ZEKE spectrum. Of note is that the spectra are structured with a clean baseline, except for the spectrum via $29^{2}$, which is weak, and the baseline is noisy from the ionization laser intensity variation.

Figure 7: ZEKE spectra for $p$ Xyl recorded at different excitation wavenumbers in the region $770-810$ $\mathrm{cm}^{-1}$ - see section of REMPI spectrum shown - which covers the $9^{1}$ transition. Corresponding letters label the excitation position on the REMPI spectrum and each ZEKE spectrum. A significant rise in the baseline and general deterioration of the structure is evident in some of the spectra - see text for discussion.

Figure 8: ZEKE spectra for $p X y l$ recorded at different excitation wavenumbers in the region 1140-1230 $\mathrm{cm}^{-1}$ - see section of REMPI spectrum shown - which covers the $9^{1}$ transition. Corresponding letters label the excitation position on the REMPI spectrum and each ZEKE spectrum. A significant rise in the baseline and general deterioration of the structure is evident in most of the spectra - see text for discussion.

Figure 9: Density of state plots showing the vibrational levels for the four subject molecule, each point in the plot represents the number of vibrations within a $10 \mathrm{~cm}^{-1}$ window. It is seen (see text) that the build-up of vibrational levels is relatively low and erratic and fairly consistent between the molecules.

Figure 10: Density of state plots showing the vibrational and vibtor (for $p \mathrm{FT}$ and $p \mathrm{Xyl}$ ) levels for the four subject molecules - note the different $y$-axis scales for the latter. (Each point in the plot represents the number of vibrations within a $10 \mathrm{~cm}^{-1}$ window.) Although the build-up of vibrational levels is relatively low and fairly consistent across the molecules (see Figure 9), the addition of the vibtor levels causes a very significant increase. To low wavenumber the erratic build-up of states is evident, and this is particularly marked for the vibrational levels (see also Figure 9).

Figure 11: Comparison of the density of states build-up with the REMPI spectra for the four subject molecules, for levels accessible from "cold" levels - see test. The sparseness of the DOS for $p$ CIFB and pDFB makes it clear why just occasional Fermi resonances occur at low wavenumber, while interactions are much more widespread for $p \mathrm{FT}$ and particularly $p$ Xyl. 
Figure 1

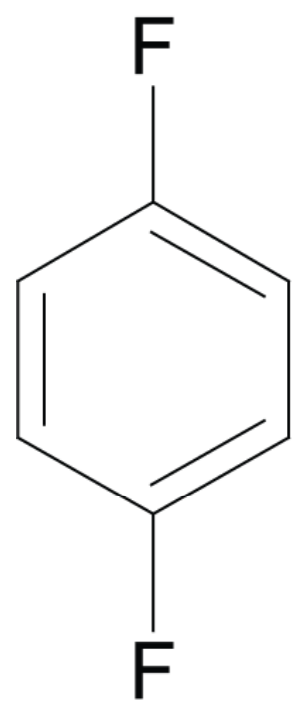

(a) $p \mathrm{DFB}$

$D_{2 h}$

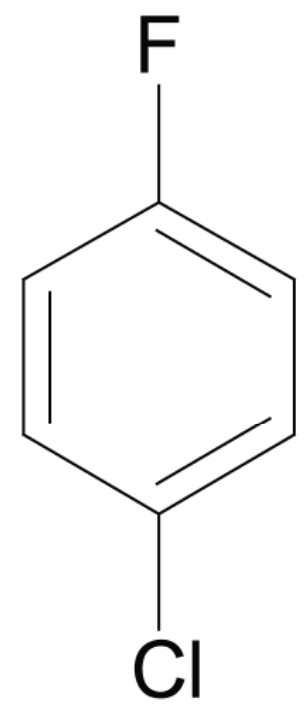

(c) $p \mathrm{ClFB}$

$C_{2 v}$

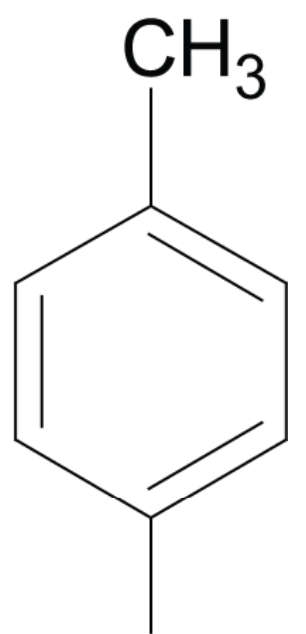

$\mathrm{CH}_{3}$

(b) $p \mathrm{Xyl}$

$D_{2 h} / G_{72}$

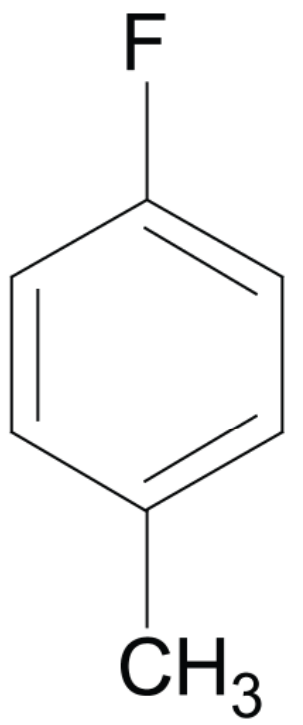

(d) $p \mathrm{FT}$

$C_{2 v} / G_{12}$ 
Figure 2

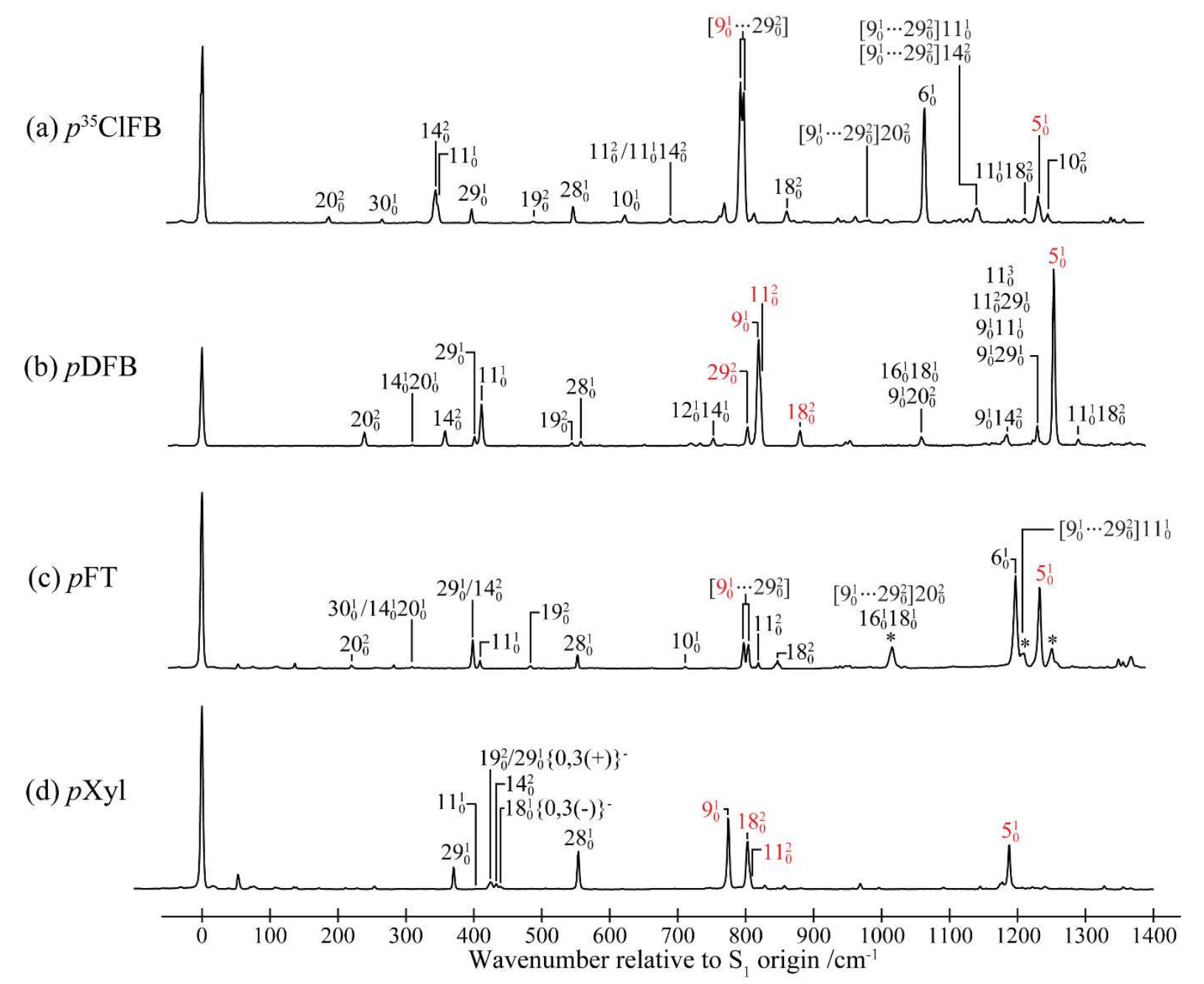




\section{Figure 3}

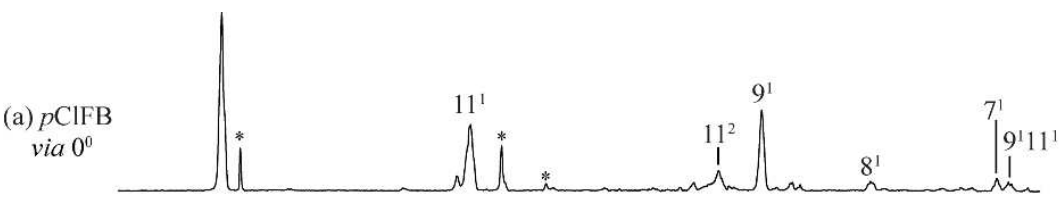

(b) $p \mathrm{DFB}$
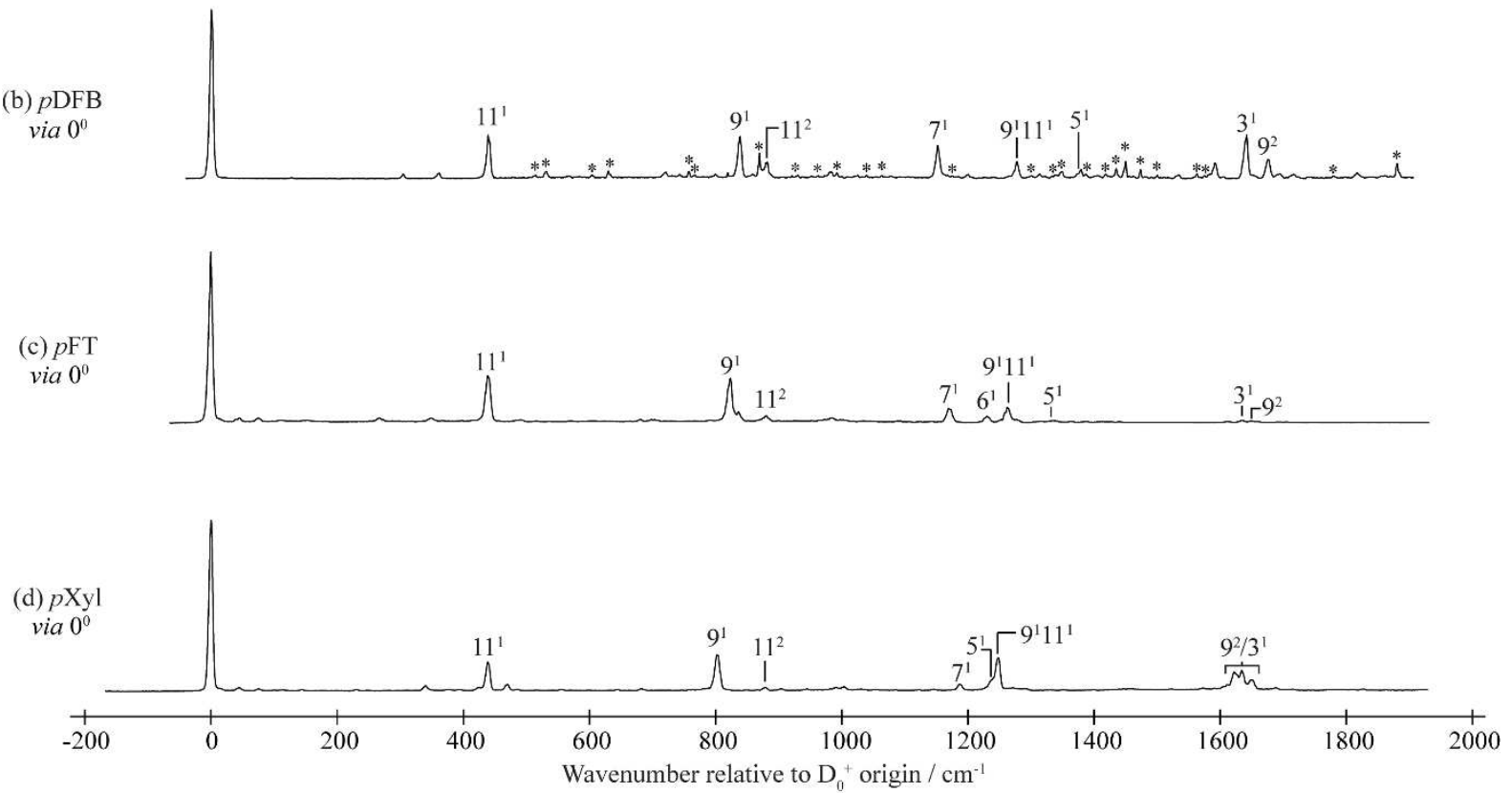


\section{Figure 4}

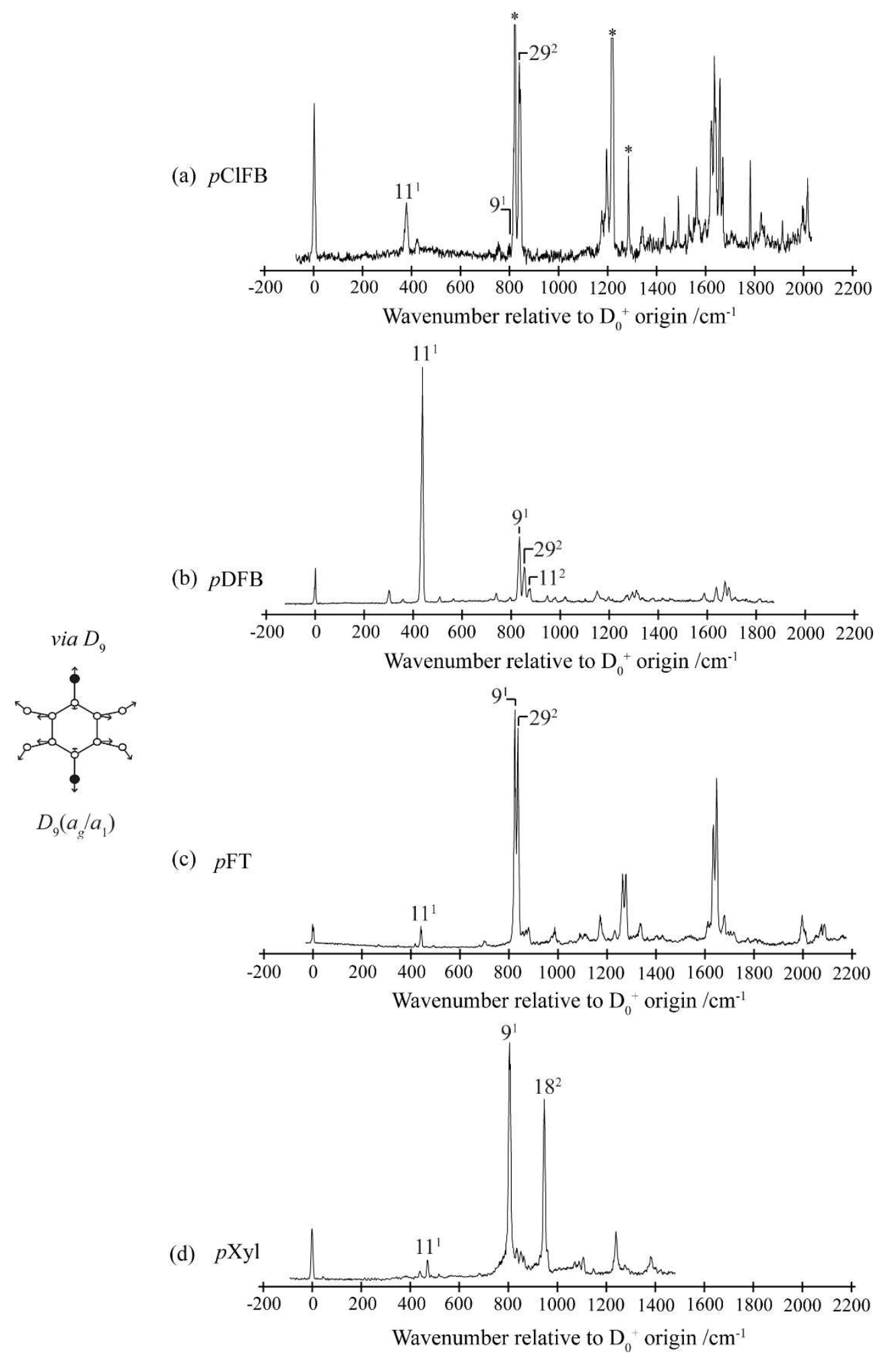




\section{Figure 5}

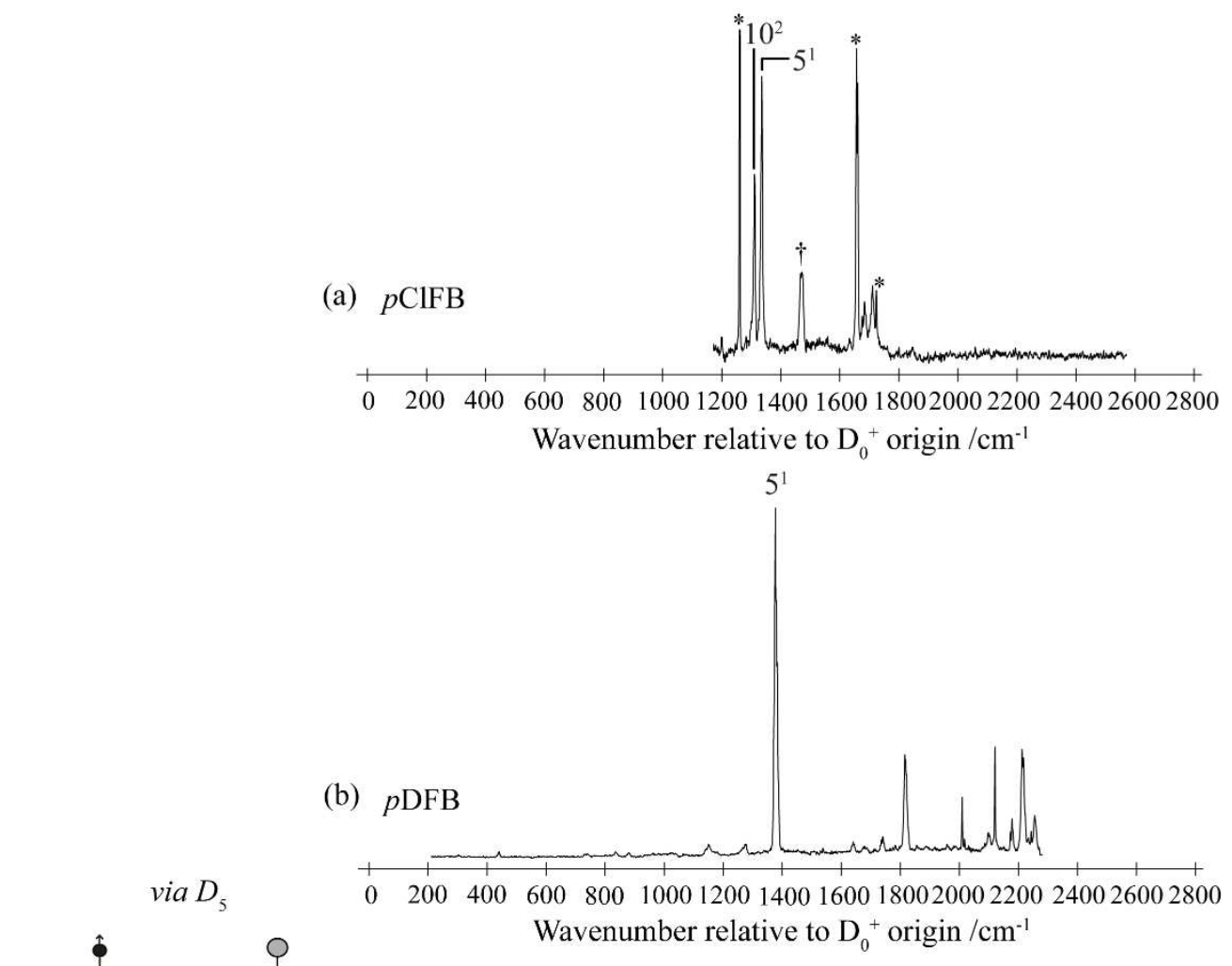

(c) $p \mathrm{FT}$

$0 \quad 200400600 \quad 8001000120014001600180020002200240026002800$

Wavenumber relative to $\mathrm{D}_{0}^{+}$origin $/ \mathrm{cm}^{-1}$

(d) $p \mathrm{Xyl}$

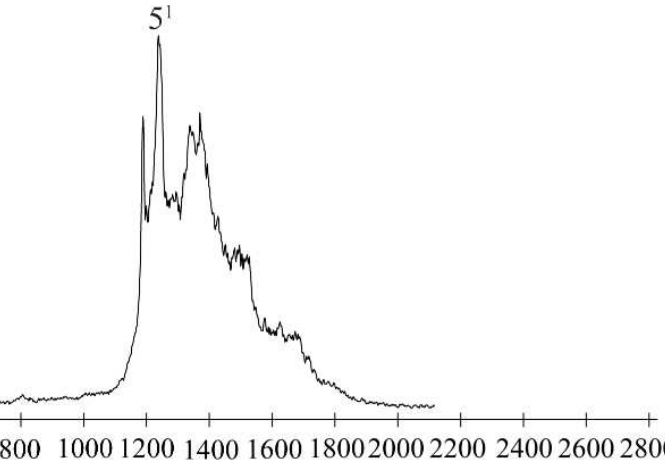

Wavenumber relative to $\mathrm{D}_{0}^{+}$origin $/ \mathrm{cm}^{-1}$ 
Figure 6

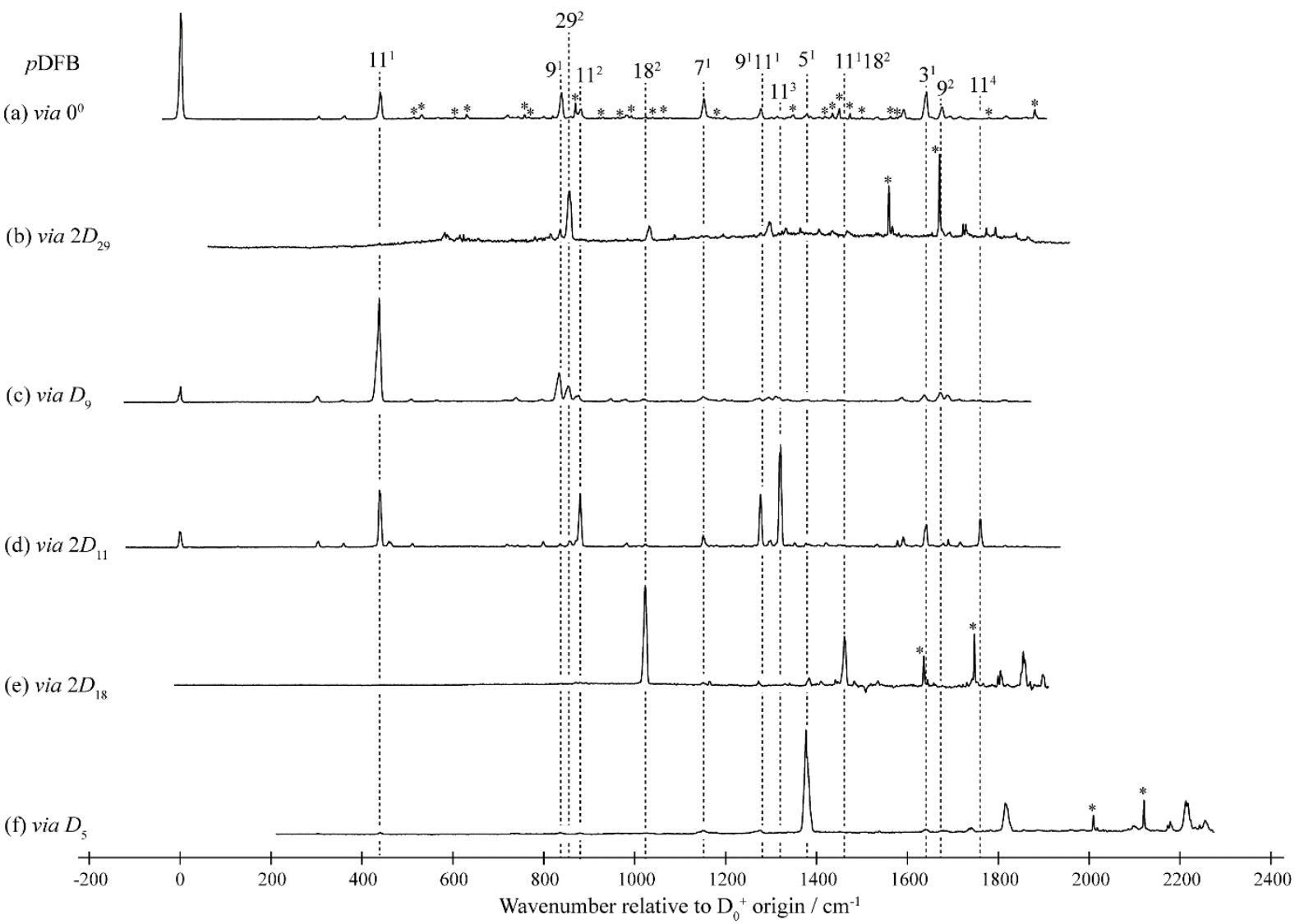




\section{Figure 7}

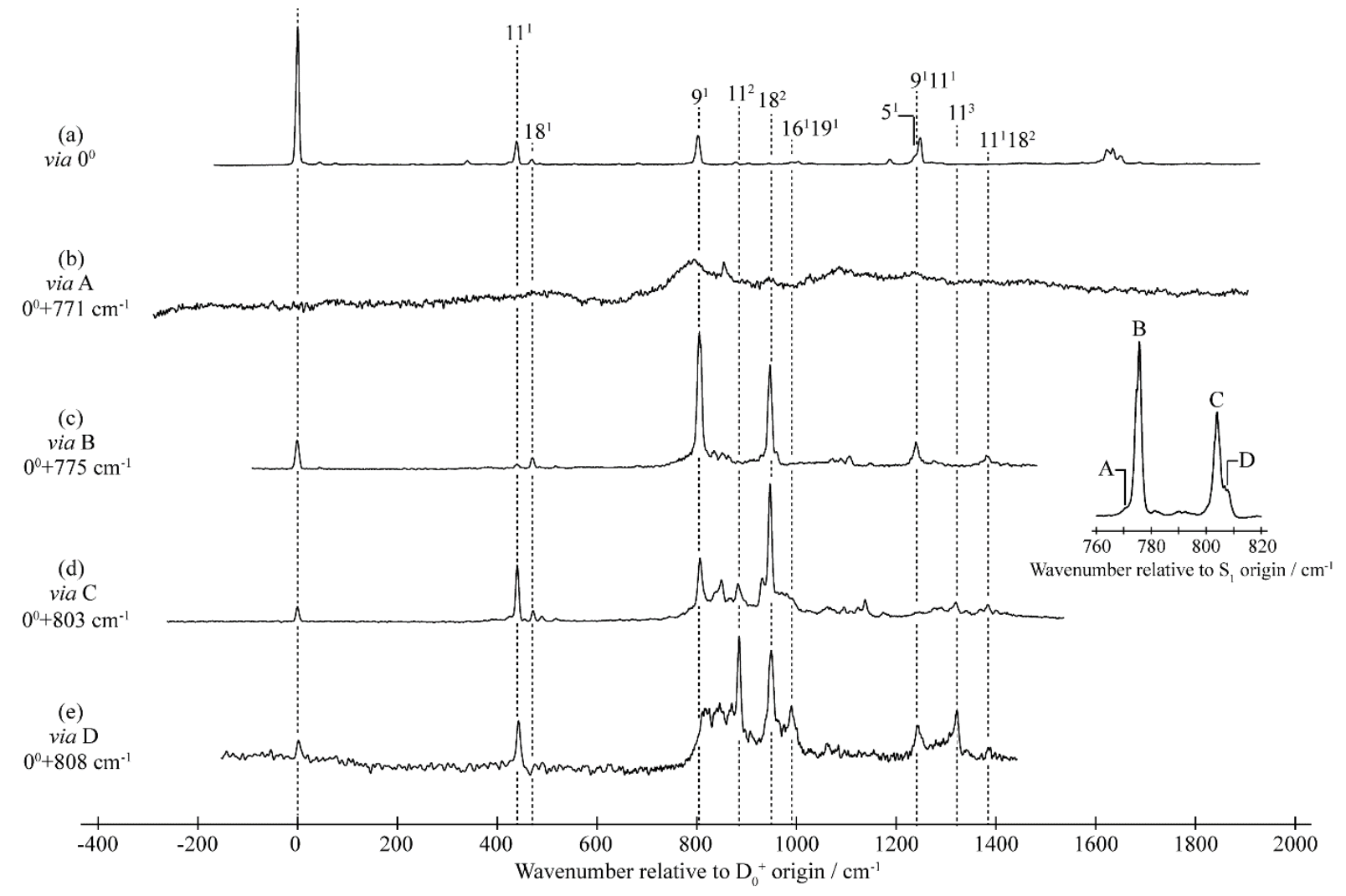




\section{Figure 8}

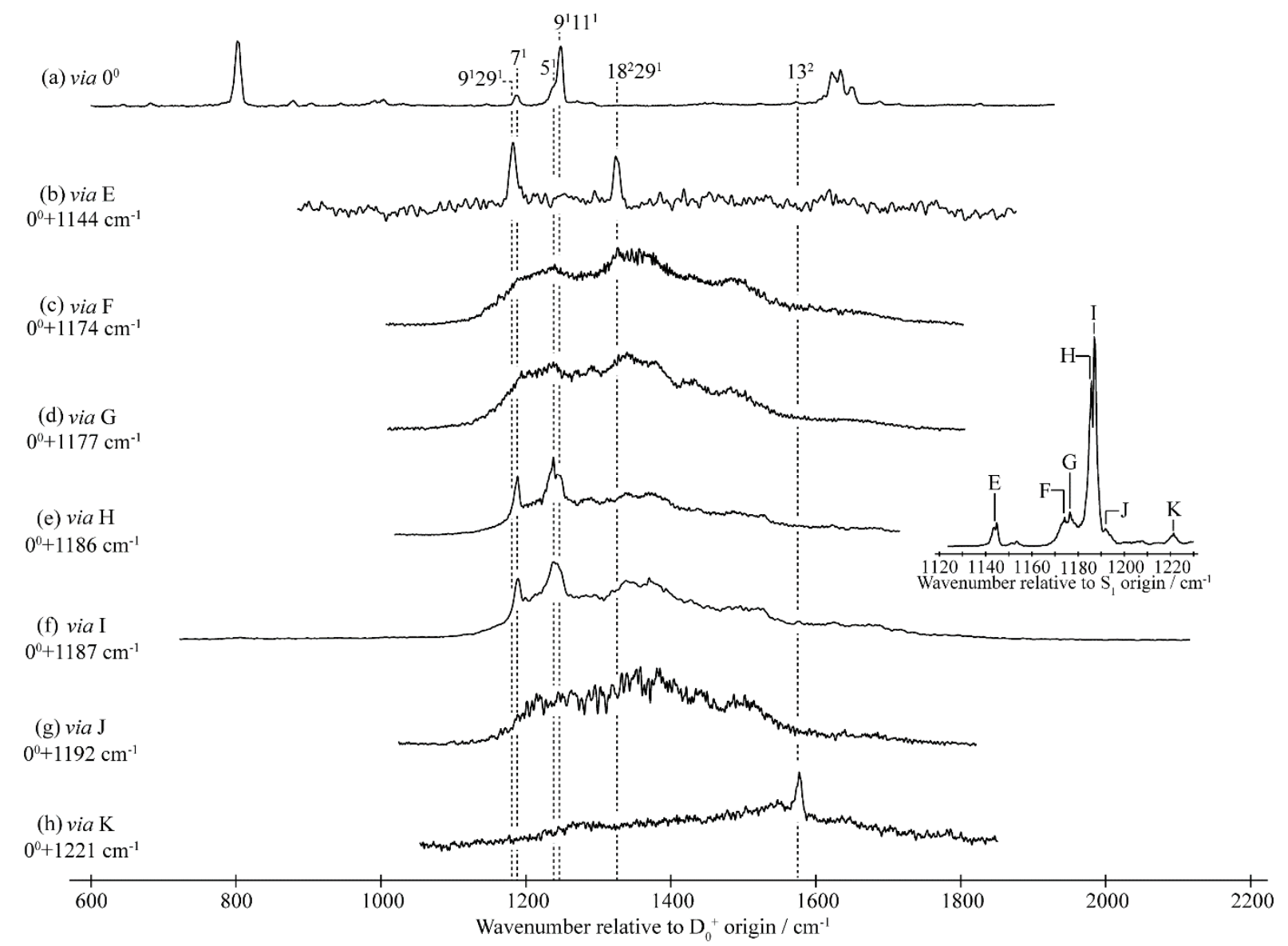


Figure 9
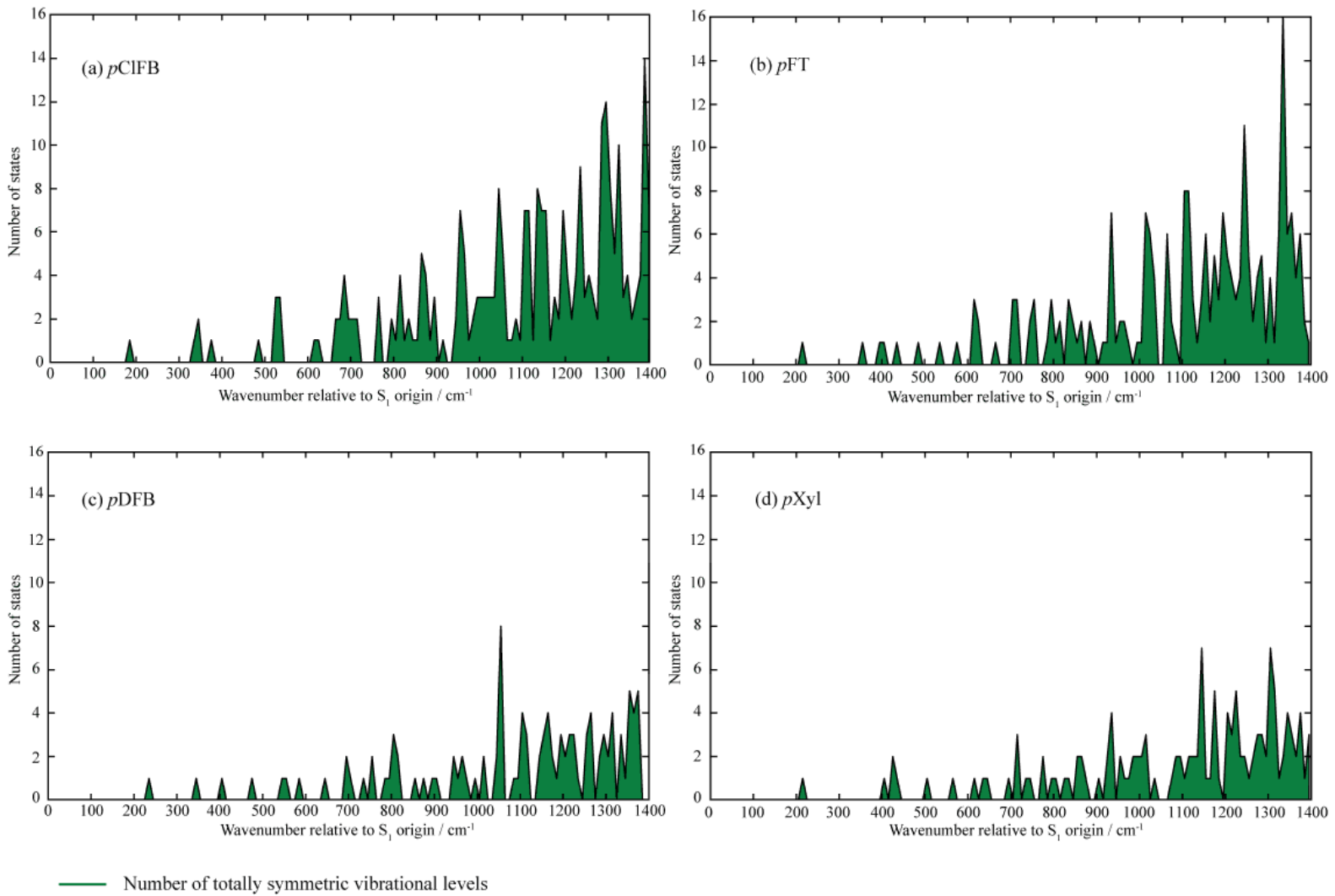
Figure 10
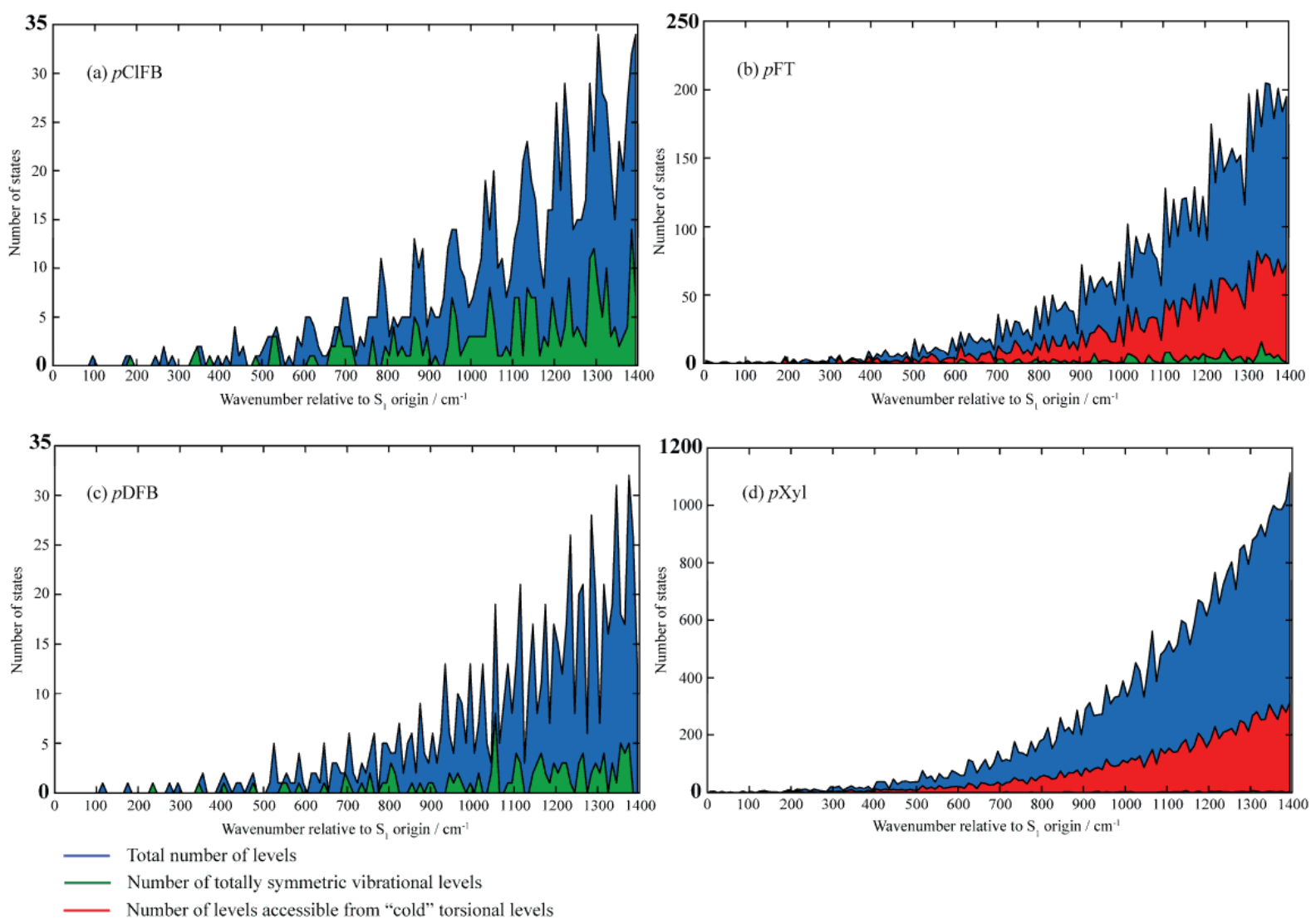

Number of levels accessible from "cold" torsional levels 


\section{Figure 11}

Number of levels

Number of totally symmetric vibrational levels

Number of levels accessible from "cold" torsional levels
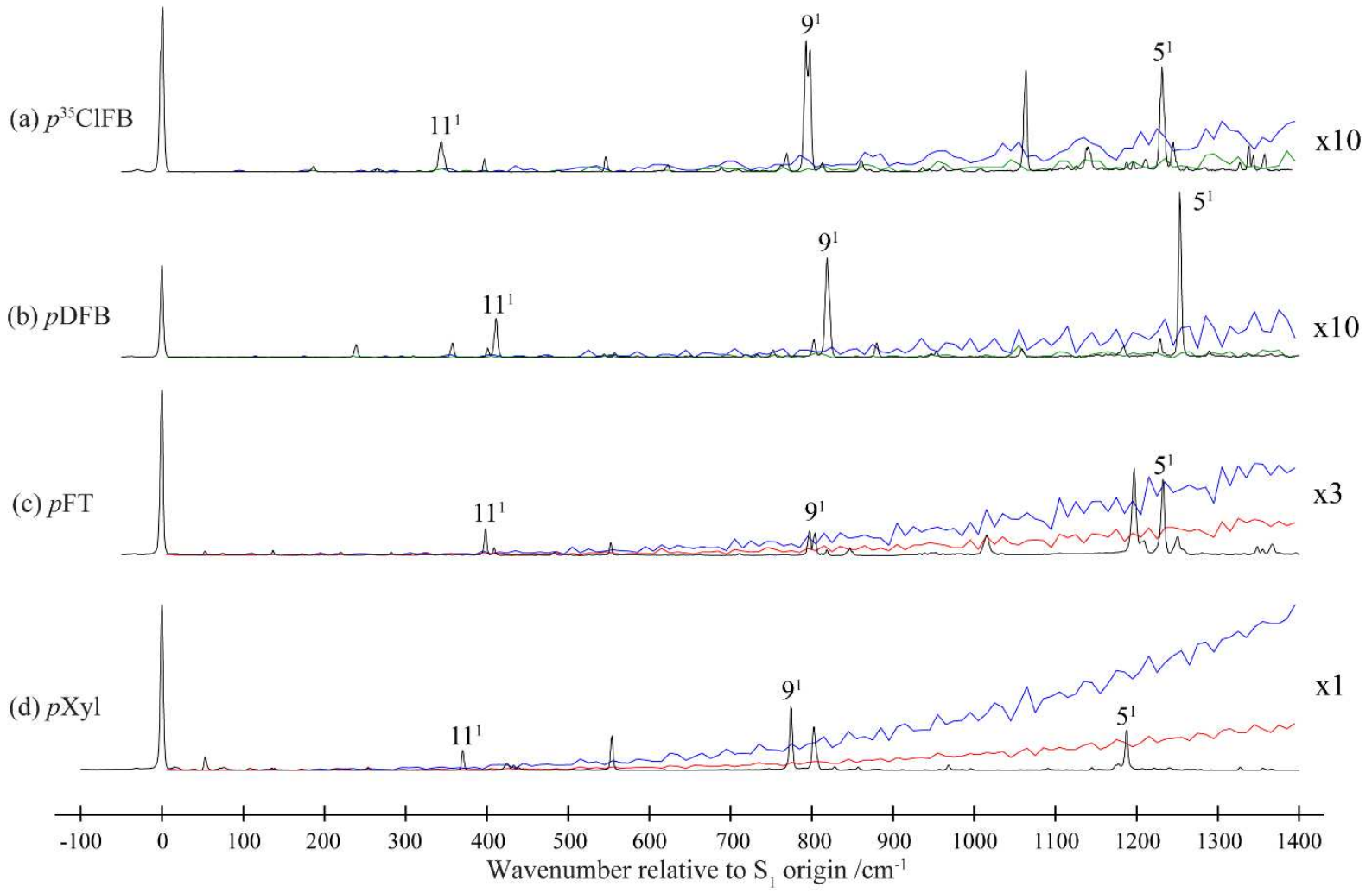


\section{References}

${ }^{1}$ G. M. Roberts and V. G. Stavros in Ultrafast Phenomena in Molecular Sciences, Ed. R. de Nalda and L. Bañares (Springer, Dordrecht, 2014).

${ }^{2}$ Y. He, C. Wu, and W. Kong, J. Phys. Chem. A, 2003, 107, 5145-5148.

${ }^{3}$ C. Skinnerup Byskov, F. Jensen, T. J. D. Jørgensen, and S. Brøndsted Nielsen, Phys. Chem. Chem. Phys., 2014, 16, 15831-15838.

${ }^{4}$ P. M. Felker and A. H. Zewail, J. Chem. Phys., 1985, 82, 2961-2974.

${ }^{5}$ P. M. Felker and A. H. Zewail, J. Chem. Phys., 1985, 82, 2975-2993.

${ }^{6}$ P. M. Felker and A. H. Zewail, J. Chem. Phys., 1985, 82, 2994-3002.

7 P. M. Felker, W. R. Lambert and A. H. Zewail, J. Chem. Phys., 1985, 82, 3003-3010.

${ }^{8}$ D. J. Nesbitt and R. W. Field, J. Phys. Chem., 1996, 100, 12735-12756.

${ }^{9}$ D. Boyall and K. L. Reid, Chem. Soc. Rev., 1997, 26, 223-232.

${ }^{10}$ K. L. Reid, Int. Rev. Phys. Chem., 2008, 27, 607-628.

${ }^{11}$ K. K. Lehmann, G. Scoles and B. H. Pate, Annu. Rev. Phys. Chem., 1994, 45, 241-274.

12 J. C. Keske and B. H. Pate, Annu. Rev. Phys. Chem., 2000, 51, 323-353.

${ }^{13}$ E. R. Gonzaga, Am. J. Clin. Dermat., 2009, 10 Suppl. 1, 19-24.

${ }^{14}$ D. R. Killalea and A. L. Utz, Phys. Chem. Chem. Phys., 2013, 15, 20545.

${ }^{15}$ See, for example: F. Di Giacomo, J. Chem. Ed., 2015, 92, 476.

${ }^{16}$ S. Yan, Y.-T. Wu, B. Zhang, X.-F. Yue and K. Liu, Science, 2007, 316, 1723.

17 J. C. Polanyi, Acc. Chem. Res., 1972, 5, 161.

${ }^{18}$ N. D. N. Rodrigues, M. Staniforth and V. G. Stavros, Proc. Roy. Soc. A, 2016, 472, 20160677.

${ }^{19}$ L. A. Baker, B. Marchetti, T. N. V. Karsili, V. G. Stavros and M. N. R. Ashfold, Chem. Soc. Rev., 2017, 46, 3770-3791.

${ }^{20}$ A. D. G. Nunn, R. S. Minns, R. Spesyvtsev, M. J. Bearpark, M. A. Robb and H. H Fielding, Phys. Chem. Chem. Phys., 2010, 12, 15751-15759.

${ }^{21}$ G. A. King, T. A. A. Oliver, R. N. Dixon and M. N. R. Ashfold, Phys. Chem. Chem. Phys. 14, 3338 (2012).

22 J. R. Gascooke and W. D. Lawrance, J. Chem. Phys., 2013, 138, 134302.

23 J. A. Davies, A. M. Green, and K. L. Reid, Phys. Chem. Chem. Phys., 2010, 12, 9872-9883.

${ }^{24}$ J. A. Davies, A. M. Green, A. M Gardner, C. M. Withers, T. G. Wright, and K. L. Reid, Phys. Chem. Chem. Phys., 2014, 16, 430-443.

25 J. R. Gascooke, E. A. Virgo, and W. D. Lawrance, J. Chem. Phys., 2015, 143, 044313. 
${ }^{26}$ J. A. Davies and K. L. Reid, Phys. Rev. Lett., 2012, 109, 193004.

${ }^{27}$ E. A. Virgo, J. R. Gascooke and W. D. Lawrance, J. Chem. Phys., 2014, 140, 154310.

${ }^{28}$ D. A. Dolson, C. S. Parmenter and B. M. Stone, Chem. Phys. Lett. 81, 360 (1981).

${ }^{29}$ A. A. Makarov, A. L. Malinovsky and E. A. Ryabov, Physics-Uspekhi, 2012, 55, 977.

${ }^{30}$ C. J Hammond, V. L. Ayles, D. Bergeron, K. L. Reid and T. G. Wright, J. Chem. Phys., 2006, 125, 124308.

${ }^{31}$ C. S. Parmenter and B. M. Stone, J. Chem. Phys., 1986, 84, 4710.

${ }^{32}$ Q. Ju, C. S. Parmenter, T. A. Stone and Z.-Q. Zhao, Isr. J. Chem., 1997, 37, 379.

33 J. S. Baskin, T. S. Rose, and A. H. Zewail, J. Chem. Phys., 1988, 88, 1458.

${ }^{34}$ D. B. Moss and C, S, Parmenter, J. Chem. Phys., 1993, 98, 6897.

${ }^{35}$ D. B. Moss, C. S. Parmenter and G. E. Ewing, J. Chem. Phys., 1987, 86, 51.

${ }^{36}$ Z.-Q. Zhao, C. S. Parmenter, D. B. Moss, A. J. Bradley, A. E. W. Knight, and K. G. Owens, J. Chem. Phys., 1992, 96, 6362.

${ }^{37}$ Z.-Q. Zhao, PhD Thesis, Indiana University (1992).

${ }^{38}$ Z.-Q. Zhao and C. S. Parmenter, Mode Selective Chemistry (Kluwer, 1991) Eds. J. Jortner, R. D. Levine, and B. Pullman. Jerusalem Symp. Quant. Chem. Biochem., 1991, 24, 127.

${ }^{39}$ A. M. Gardner, W. D. Tuttle, L. E. Whalley, and T. G. Wright, Chem. Sci., 2018, 9, 2270.

${ }^{40}$ R. S. von Benten, Y. Liu and B. Abel, J. Phys. Chem. A, 2010, 114, 11522.

${ }^{41}$ J. Aßmann, M. Kling and B. Abel, Angew. Chem. Int. Ed. Engl., 2003, 42, 2226.

${ }^{42}$ D. P. Mukhopadhyay, S. Biswas and T. Chakraborty, J. Phys. Chem. A, 2016, 120, 9159.

${ }^{43}$ D. P. Mukhopadhyay, S. Biswas and T. Chakraborty, Chem. Phys. Lett., 2017, 674, 71.

44 J. A. Davies, L. E. Whalley and K. L. Reid, Phys. Chem. Chem. Phys., 2017, 19, 5051.

${ }^{45}$ G. A. Bethardy, X. Wang and D. S. Perry, Can. J. Chem., 1994, 72, 652.

${ }^{46}$ D. S. Perry, G. A. Bethardy and X. Wang, Ber. Bunsenges. Phys. Chem., 1995, 99, 530.

${ }^{47}$ C. C. Martens and W. P. Reinhardt, J. Chem. Phys., 1990, 93, 5621.

${ }^{48}$ L. W. Peng, B. W. Keelan, D. H. Semmes and A. H. Zewail, J. Phys. Chem., 1988, 92, 554.

${ }^{49}$ M. Hippler, R. Pfab, and M. Quack, J. Phys. Chem. A, 2003, 107, 10743.

${ }^{50}$ R. S. von Benten, Y. Liu, and B. Abel, J. Chem. Phys., 2010, 133, 134306.

${ }^{51}$ A. Andrejeva, A. M. Gardner, W. D. Tuttle and T. G. Wright, J. Molec. Spectrosc., 2016, 321, 28-49.

${ }^{52}$ V. L. Ayles, C. J. Hammond, D. E. Bergeron, O. J. Richards and T. G. Wright, J. Chem. Phys., 2007, 126, 244304.

${ }^{53}$ A. M. Gardner, W. D. Tuttle, L. Whalley, A. Claydon, J. H. Carter and T. G. Wright, J. Chem. Phys., 2016, 145, 124307.

${ }^{54}$ W. D. Tuttle, A. M. Gardner, L. E. Whalley, and T. G. Wright, J. Chem. Phys., 2017, 146, 244310. 
${ }^{55}$ W. D. Tuttle, A. M. Gardner and T. G. Wright, Chem. Phys. Lett., 2017, 684, 339.

${ }^{56}$ D. J. Kemp, L. E. Whalley, W. D. Tuttle, A. M. Gardner, B. T. Speake and T. G. Wright, Phys. Chem. Chem. Phys., 2018, DOI: 10.1039/C8CP01274A

${ }^{57}$ A. M. Gardner, W. D. Tuttle, P. Groner, and T. G. Wright, J. Chem. Phys., 2017, 146, 124308.

${ }^{58}$ W. D. Tuttle, A. M. Gardner, K. B. O'Regan, W. Malewicz, and T. G. Wright, J. Chem. Phys., 2017, $146,124309$.

${ }^{59}$ D. Rieger, G. Reiser, K. Müller-Dethlefs, and E. W. Schlag, J. Phys. Chem., 1992, 96, 12.

${ }^{60}$ G. Reiser, D. Rieger, T. G. Wright, K. Müller-Dethlefs and E. W. Schlag, J. Phys. Chem., 1993, 97, 4335.

${ }^{61}$ S. D. Gamblin, S. E. Daire, J. Lozeille, and T. G. Wright, Chem. Phys. Lett., 2000, 325, 232.

${ }^{62}$ X. Zhang, J. M. Smith, and J. L. Knee, J. Chem. Phys., 1992, 97, 2843.

${ }^{63}$ P. M. Felker and A. H. Zewail, Adv. Chem. Phys., 1988, 70, 265.

${ }^{64}$ D. R. Demmer, J. W. Hager, G. W. Leach and S. C. Wallace, Chem. Phys. Lett., 1987, 136, 329.

${ }^{65}$ W. D. Lawrance and J. R. Gascooke, Pervasive interactions between methyl torsion and low frequency vibrations in $\mathrm{S}_{0}$ and $\mathrm{S}_{1}$ p-fluorotoluene. J. Chem. Phys. (submitted).

${ }^{66}$ E. Sekreta, K. S. Viswanathan, and J. P. Reilly, J. Chem. Phys., 1989, 90, 5349.

${ }^{67}$ C. H. Kwon, H. L. Kim and M. S. Kim, J. Chem. Phys., 2003, 118, 6327.

${ }^{68}$ R. A. Coveleskie and C. S. Parmenter, J. Molec. Spectrosc., 1981, 86, 86.

${ }^{69}$ A. E. W. Knight and S. H. Kable, J. Chem. Phys., 1988, 89, 7139.

${ }^{70}$ B. T. Darling and D. M. Denison, Phys. Rev., 1940, 57, 128. 